\title{
LYAPUNOV EXPONENTS FOR PRODUCTS OF MATRICES AND MULTIFRACTAL ANALYSIS. PART II: GENERAL MATRICES
}

\author{
DE-JUN FENG
}

\begin{abstract}
We continue the study in $[11,14]$ on the upper Lyapunov exponents for products of matrices. Here we consider general matrices. In general, the variational formula about Lyapunov exponents we obtained in part I does not hold in this setting. Anyway we focus our interest on a special case where the matrix function $M(x)$ takes finite values $M_{1}$, $\ldots, M_{m}$. In this case we prove the variational formula under an additional irreducibility condition. This extends a previous result of the author and Lau [14]. As an application, we prove a new multifractal formalism for a certain class of self-similar measures on $\mathbb{R}$ with overlaps. More precisely, let $\mu$ be the self-similar measure on $\mathbb{R}$ generated by a family of contractive similitudes $\left\{S_{j}=\rho x+b_{j}\right\}_{j=1}^{\ell}$ which satisfies the finite type condition. Then we can construct a family (finite or countably infinite) of closed intervals $\left\{I_{j}\right\}_{j \in \Lambda}$ with disjoint interiors, such that $\mu$ is supported on $\bigcup_{j \in \Lambda} I_{j}$ and the restricted measure $\left.\mu\right|_{I_{j}}$ of $\mu$ on each interval $I_{j}$ satisfies the complete multifractal formalism. Moreover the dimension spectrum $\operatorname{dim}_{H} E_{\left.\mu\right|_{I_{j}}}(\alpha)$ is independent of $j$.
\end{abstract}

\section{INTRODUCTION}

The present paper is a continuation of our work in $[11,14]$ for studying the upper Lyapunov exponents for products of matrices. Here we consider general matrices. An application will be given to multifractal analysis of self-similar measures with overlaps.

First we recall some basic notation. Let $\sigma$ be the shift map on $\Sigma=\{1,2, \ldots, m\}^{\mathbb{N}}(m \geq 2$ an integer). Endow $\Sigma$ with the metric $d(x, y)=m^{-n}$ for $x=\left(x_{j}\right)_{j=1}^{\infty}$ and $y=\left(y_{j}\right)_{j=1}^{\infty}$ where $n$ is the largest integer such that $x_{j}=y_{j}(1 \leq j \leq n)$. Let $M$ be a continuous function defined on $\Sigma$ taking values in the set of $d \times d$ matrices with non-negative entries. The upper Lyapunov exponent $\lambda_{M}(x)$ of $M$ at $x$ is defined by

$$
\lambda_{M}(x)=\lim _{n \rightarrow \infty} \frac{1}{n} \log \left\|M(x) M(\sigma x) \ldots M\left(\sigma^{n-1} x\right)\right\|,
$$

when the limit exists. Here $\|\cdot\|$ denotes the matrix norm defined by $\|A\|:=\mathbf{1}^{\tau} A \mathbf{1}$, where $\mathbf{1}$ is the $d$-dimensional column vector each coordinate of which is 1 . The pressure function of $M$ is defined by

$$
P_{M}(q)=\lim _{n \rightarrow \infty} \frac{1}{n} \log \sum_{\omega \in \Sigma_{n}} \sup _{x \in[\omega]}\left\|M(x) M(\sigma x) \ldots M\left(\sigma^{n-1} x\right)\right\|^{q}, \quad q \in \mathbb{R},
$$

Key words and phrases. Matrix products, Lyapunov exponents, Hausdorff dimensions, Packing dimensions, Entropies, Pressure functions, Multifractals, Self-similar measures.

The author was partially supported by the direct grant and RGC grants (Projects 400706, 401008) and in CUHK, Fok Ying Tong Education Foundation and NSFC (Grant 10571100). 
where $\Sigma_{n}$ denotes the set of all words of length $n$ over $\{1, \ldots, m\}$; for $\omega=\omega_{1} \ldots \omega_{n} \in \Sigma_{n}$, $[\omega]$ denotes the cylinder set $\left\{x=\left(x_{j}\right)_{j=1}^{\infty} \in \Sigma: x_{j}=\omega_{j}, 1 \leq j \leq n\right\}$. The limit in the above definition always exists for $q>0$. This can be shown by a subadditive argument. With some additional conditions on $M$ (e.g., $M$ is strictly positive), the limit exists for $q \in \mathbb{R}$. Let $\mathcal{M}_{\sigma}(\Sigma)$ be the set of all $\sigma$-invariant Borel probability measures on $\Sigma$. The matrix function $M$ induces a map $M_{*}: \mathcal{M}_{\sigma}(\Sigma) \rightarrow \mathbb{R} \cup\{-\infty\}$ given by

$$
M_{*}(\mu)=\lim _{n \rightarrow \infty} \frac{1}{n} \int \log \left\|M(y) M(\sigma y) \ldots M\left(\sigma^{n-1} y\right)\right\| d \mu(y), \quad \mu \in \mathcal{M}_{\sigma}(\Sigma) .
$$

The limit exists because of the subadditivity of the integral. In [18] Furstenberg and Kesten proved that for each ergodic measure $\mu$ on $\Sigma$,

$$
\lambda_{M}(x)=M_{*}(\mu), \quad \mu \text { a.s. } x \in \Sigma .
$$

To study the point-wise property of $\lambda_{M}(\cdot)$ more delicately, we define

$$
L_{M}=\left\{\alpha \in \mathbb{R}: \alpha=\lambda_{M}(x) \text { for some } x \in \Sigma\right\}
$$

and

$$
E_{M}(\alpha)=\left\{x \in \Sigma: \lambda_{M}(x)=\alpha\right\} \quad\left(\alpha \in L_{M}\right) .
$$

The following theorem was proved in [11]:

Theorem A ([11]). Suppose $M$ is a continuous function defined on $\Sigma$ taking values in the set of $d \times d$ matrices with strictly positive entries. Then $L_{M}$ is an interval and for any $\alpha \in L_{M}$,

$$
\begin{aligned}
& \operatorname{dim}_{H} E_{M}(\alpha)=\operatorname{dim}_{P} E_{M}(\alpha) \\
= & \frac{1}{\log m} \inf _{q \in \mathbb{R}}\left\{-\alpha q+P_{M}(q)\right\} \\
= & \frac{1}{\log m} \sup \left\{h(\mu): \mu \in \mathcal{M}_{\sigma}(\Sigma), M_{*}(\mu)=\alpha\right\},
\end{aligned}
$$

where $\operatorname{dim}_{H}$ and $\operatorname{dim}_{P}$ denote the Hausdorff dimension and the packing dimension, respectively, and $h(\mu)$ denotes the measure-theoretic entropy of $\mu$. Moreover, $\operatorname{dim}_{H} E_{M}(\alpha)$ is a concave and continuous function of $\alpha$ on $L_{M}$.

Theorem A extends the corresponding results for the Birkhoff averages of scalar functions, for which one is referred to, e.g., [1, 2, 3, 9, 10, 16, 35, 38, 40, 41, 47]. However since Theorem A depends very much on the positivity of $M$, for certain purposes of application (e.g., the multifractal analysis of self-similar measures with overlaps) we would like to consider the case that the matrix function $M$ is only assumed to be non-negative. However under this general non-negative assumption, the result of Theorem A is no longer valid. The following is a counter-example.

Example 1.1 Set $\Sigma=\{1,2,3,4\}^{\mathbb{N}}$. Let the matrix function $M(x)$ on $\Sigma$ be defined as $M(x)=M_{x_{1}}$ for $x=\left(x_{j}\right)_{j=1}^{\infty}$, where $M_{i}(1 \leq i \leq 4)$ are four diagonal matrices given by

$$
M_{1}=M_{2}=\operatorname{diag}(1,2,0,0), \quad M_{3}=\operatorname{diag}(1,0,3,0), M_{4}=\operatorname{diag}(1,0,0,4) .
$$

It is easily checked that

$$
P_{M}(q)= \begin{cases}q \log 4, & \text { if } q \geq 1, \\ \log 4, & \text { otherwise. }\end{cases}
$$

However $L_{M}=\{0, \log 2, \log 3, \log 4\}$, and

$$
\operatorname{dim}_{H} E_{M}(\log 3)=0<1-\frac{\log 3}{\log 4}=\frac{1}{\log 4} \inf _{q \in \mathbb{R}}\left\{-q \log 3+P_{M}(q)\right\} .
$$


For the above reason, we shall impose more conditions on $M$ :

(H1) $M(x)=M_{i}$ if $x \in[i], i=1, \ldots, m$;

(H2) $M$ is irreducible in the following sense: there exists $r>0$ such that

$$
\sum_{k=1}^{r}\left(\sum_{i=1}^{m} M_{i}\right)^{k}>\mathbf{0}
$$

In this case, the pressure function $P_{M}(q)$ can be defined by

$$
P_{M}(q)=\lim _{n \rightarrow \infty} \frac{1}{n} \log \sum_{J \in \mathcal{N}_{n}}\left\|M_{J}\right\|^{q}, \quad \forall q \in \mathbb{R},
$$

where $\mathcal{N}_{n}=\left\{J \in \Sigma_{n}: M_{J} \neq \mathbf{0}\right\}$ and $M_{J}=M_{j_{1}} \cdots M_{j_{n}}$. It was proved in [14] that the limit exists for any $q \in \mathbb{R}$ (we include a proof in section 2 for the convenience of the reader). The first result of this paper is the following:

Theorem 1.1. Suppose that $M$ is a function on $\Sigma$ taking values in the set of all $d \times d$ non-negative matrices. Assume $M$ satisfies the conditions (H1) and (H2). Then $L_{M}$ is an interval and for any $\alpha \in L_{M}$,

$$
\operatorname{dim}_{H} E_{M}(\alpha)=\operatorname{dim}_{P} E_{M}(\alpha)=\frac{1}{\log m} \inf _{q \in \mathbb{R}}\left\{-\alpha q+P_{M}(q)\right\} .
$$

Moreover $\operatorname{dim}_{H} E_{M}(\alpha)$ is a concave and continuous function of $\alpha$ on $L_{M}$.

Under the setting of this theorem, some partial results were obtained by Feng and Lau in [14]. It was shown that for each $q>0$, there exists a unique ergodic probability measure $\mu_{q}$ on $\Sigma$ satisfying the following Gibbs property:

$$
C^{-1} \leq \frac{\mu_{q}([J])}{\exp \left(-n P_{M}(q)\right) \cdot\left\|M_{J}\right\|^{q}} \leq C, \quad \forall n \in \mathbb{N}, \quad J \in \Sigma_{n}
$$

where $C>0$ is a constant (see [14, Theorem 3.2]). The measure $\mu_{q}$ on $\Sigma$ is called the Gibbs measure associated with $M$ and $q$. Using this Gibbs property, we proved that $P_{M}(q)$ is always differentiable on $(0, \infty)$ and $(1.6)$ holds for any $\alpha=P_{M}^{\prime}(q)$ with $q>0$ (see [14, Theorems 3.3 and 3.4]). Unfortunately, the existence of the Gibbs measure can not be extended to the case $q<0$; actually we can construct a matrix function $M$ satisfying (H1) and (H2) such that $P_{M}(q)$ is not differentiable at some point $q<0$ (see Example 6.6). Hence some new idea is needed to prove (1.6) for all $\alpha \in L_{M}$.

Now we outline the idea for proving Theorem 1.1. Under the condition of the theorem, there is a constant $C>0$ such that for any $I \in \Sigma_{n}$ and $J \in \Sigma_{\ell}$ there exists $K \in \bigcup_{k=1}^{r} \Sigma_{k}$ such that $\left\|M_{I K J}\right\| \geq C\left\|M_{I}\right\|\left\|M_{J}\right\|$. Using this property and modifying our proof in [11] delicately, we set up a formal formula for $\operatorname{dim}_{H} E_{M}(\alpha)$ similar to that in [11]. To show the variational relationship between $\operatorname{dim}_{H} E_{M}(\alpha)$ and $P_{M}(q)$, we use a new idea. In fact we first give such a relationship between $\operatorname{dim}_{H} E_{M}^{(\ell)}(\alpha)$ and $P_{M}(\ell, q)(\ell=1,2, \ldots)$, where

$$
E_{M}^{(\ell)}(\alpha)=\left\{\left(x_{i}\right)_{i=1}^{\infty} \in \Sigma: \lim _{n \rightarrow \infty} \frac{1}{n \ell} \log \left(\prod_{j=1}^{n}\left\|M_{x_{(j-1) \ell+1} x_{(j-1) \ell+2} \cdots x_{j \ell}}\right\|\right)=\alpha\right\}, \quad \alpha \in \mathbb{R}
$$

and $P_{M}(\ell, q)=\frac{1}{\ell} \log \left(\Sigma_{J \in \mathcal{N}_{\ell}}\left\|M_{J}\right\|^{q}\right)$. Then we prove the theorem by showing that $\operatorname{dim}_{H} E_{M}^{(\ell)}(\alpha)$ and $P_{M}(\ell, q)$ converge to $\operatorname{dim}_{H} E_{M}(\alpha)$ and $P_{M}(q)$ respectively, as $\ell$ tends to infinity. 
We point out that an essentially identical proof shows that the result of Theorem 1.1 remains true if we remove the non-negativity assumption of $\left\{M_{i}\right\}_{i=1}^{m}$, but instead of (H2) we assume directly the more general condition (H2)': there exist a constant $C>0$ and $r \in \mathbb{N}$ such that for any $I \in \Sigma_{n}$ and $J \in \Sigma_{\ell}$ there exists $K \in \bigcup_{k=1}^{r} \Sigma_{k}$ such that $\left\|M_{I K J}\right\| \geq C\left\|M_{I}\right\|\left\|M_{J}\right\|$. Here we use the standard matrix norm. For instance, this assumption (H2)' is fulfilled if the family $\left\{M_{i}\right\}_{i=1}^{m}$ of $d \times d$ complex matrices satisfies the following type of irreducibility: there is no proper non-zero linear subspace $V$ of $\mathbb{C}^{d}$ such that $M_{i}(V) \subseteq V$ for any $1 \leq i \leq m$ (cf. [6, p. 48]). For a proof, see Proposition 2.8. In this situation, the left endpoint of $L_{M}$ may be $-\infty$.

Theorem 1.1 has an important application in the multifractal analysis for a class of self-similar measures with overlaps. Actually, this is part of our original motivation for developing the multifractal theory for products of matrices. Before stating the result, we recall some notation and background. Let $\nu$ be a finite Borel measure on $\mathbb{R}^{n}$ with compact support. For $q \in \mathbb{R}$, the $L^{q}$ spectrum of $\nu$ is defined by

$$
\tau(q)=\tau(\nu, q)=\liminf \operatorname{ind}_{\delta \downarrow} \frac{\log \left(\sup \sum_{i} \nu\left(B_{\delta}\left(x_{i}\right)\right)^{q}\right)}{\log \delta},
$$

where the supremum is taken over all the families of disjoint balls $B_{\delta}\left(x_{i}\right)$ of radius $\delta$ and center $x_{i} \in \operatorname{supp}(\nu)$. Denote by

$$
L_{\nu}=\left\{\alpha \in \mathbb{R}: \lim _{\delta \downarrow 0} \frac{\log \nu\left(B_{\delta}(x)\right)}{\log \delta}=\alpha \text { for some } x \in \operatorname{supp}(\nu)\right\}
$$

and

$$
E_{\nu}(\alpha)=\left\{x \in \operatorname{supp}(\nu): \lim _{\delta \downarrow 0} \frac{\log \nu\left(B_{\delta}(x)\right)}{\log \delta}=\alpha\right\} .
$$

We say that $\nu$ satisfies the complete multifractal formalism if the following two conditions are satisfied:

(i) $L_{\nu}=\left[\alpha_{\min }, \alpha_{\max }\right]$, where

$$
\alpha_{\min }=\lim _{q \rightarrow+\infty} \frac{\tau(q)}{q}, \quad \alpha_{\max }=\lim _{q \rightarrow-\infty} \frac{\tau(q)}{q} .
$$

(ii) For any $\alpha \in L_{\nu}, \operatorname{dim}_{H} E_{\nu}(\alpha)=\inf \{\alpha q-\tau(q): q \in \mathbb{R}\}$.

For the framework of multifractal analysis of general measures, one may see, e.g., [4, 7, 8, 21, $24,25,26,37,39,42,44]$. It is well known that if $\mu$ is the self-similar measure defined by a family of contractive similitudes $\left\{S_{j}\right\}_{j=1}^{\ell}$ which satisfies the open set condition [28], $\tau(q)$ can be calculated by an explicit analytic formula and $\mu$ satisfies the complete multifractal formalism (see $[8,37])$. However, if the family $\left\{S_{j}\right\}_{j=1}^{\ell}$ does not satisfy any separation condition, it is much hard to obtain a formula for $\tau(q)$ and there are few results on the validity of the multifractal formalism. Some partial results have been obtained in the case that the family $\left\{S_{j}\right\}_{j=1}^{\ell}$ satisfies some separation conditions weaker than the open set condition. In [31] Lau and Ngai introduced a weak separation condition, which is strictly weaker than the open set condition. They proved that a partial multifractal formalism will hold under this condition, i.e., $\operatorname{dim}_{H} E_{\mu}(\alpha)=\inf \{\alpha q-\tau(q): q \in \mathbb{R}\}$ for all those $\alpha \in\left\{\tau^{\prime}(t): \quad t>0\right.$ and $\tau^{\prime}(t)$ exists $\}$. It is unknown whether $\tau$ is always differentiable for $t>0$ under the weak separation condition. Nevertheless, in [12], the author proved that $\tau(q)$ is differentiable on $(0, \infty)$ whenever the family $\left\{S_{j}\right\}_{j=1}^{\ell}$ has the same contractive ratio on $\mathbb{R}$ and satisfies the finite type condition. Recall that a family of similitudes

$$
S_{j}(x)=\rho x+b_{j}, \quad 0<\rho<1, \quad b_{j} \in \mathbb{R}, \quad j=1, \ldots, \ell
$$


satisfies the finite type condition if there is a finite set $\Gamma \subset \mathbb{R}^{+}$such that for each integer $n>0$ and any two indices $J=j_{1} \ldots j_{n}$ and $J^{\prime}=j_{1}^{\prime} \ldots j_{n}^{\prime}$,

$$
\text { either } \quad \rho^{-n}\left|S_{J}(0)-S_{J^{\prime}}(0)\right|>c \quad \text { or } \quad \rho^{-n}\left|S_{J}(0)-S_{J^{\prime}}(0)\right| \in \Gamma,
$$

where $S_{J}$ denotes the composition $S_{j_{1}} \circ \ldots \circ S_{j_{n}}$ and

$$
c=(1-\rho)^{-1}\left(\max _{1 \leq j \leq \ell} b_{j}-\min _{1 \leq i \leq \ell} b_{i}\right) .
$$

The finite type condition was introduced by Ngai and Wang [33]. It is substantially weaker than the open set condition, but a little stronger than the weak separation condition (see [34]). The finite type condition is satisfied by many interesting overlap cases, such as the Bernoulli convolutions associated with Pisot numbers. Combining the results in [12] and [31], under the finite type condition, we have $\operatorname{dim}_{H} E_{\mu}(\alpha)=\inf \{\alpha q-\tau(q): q \in \mathbb{R}\}$ for all those $\alpha \in\left\{\tau^{\prime}(t): t>0\right\}$. Recently this result was extended by Testud [49] to a special class of self-similar measures which satisfy the weak separation condition. Now a natural question arises as to whether or not the complete multifractal formalism holds in the setting of the finite type condition. To answer the question it suffices to consider the case $t<0$. Some special examples have been considered towards this direction. In [13], the author proved that for the Erdös measure $\mu$ (i.e., the Bernoulli convolution associated with the golden ratio), the function $\tau(q)$ is not differentiable at some point $q<0$. It is rather surprising. Nevertheless, the multifractal formalism (for all $\alpha \in \operatorname{int}\left(L_{\mu}\right)$ ) of such a measure is proved to be valid by Feng and Olivier [17], which extended the partial results in [30, 43]. For Bernoulli convolutions associated with other Pisot numbers, see [13] and [36]. In [27] Hu and Lau studied another interesting measure $\mu$, which is the three-fold convolution of the standard Cantor measure, and they found that the set $L_{\mu}$ is not an interval (actually it is a union of an interval and an isolated point). That is also a very strange fact (it shows that the complete multifractal formalism can fail for a self-similar measure satisfying the finite type condition). Later on this measure was extensively studied in $[15,32]$. Similarly its $L^{q}$-spectrum $\tau(q)$ has a non-differentiable point in $(-\infty, 0)$ [32], whilst an adjusted multifractal formalism still holds [15]. More interesting examples with similar phenomena were found and studied by Shmerkin [46] and Testud [49]. For a general self-similar measure $\mu$ on $\mathbb{R}$ satisfying the finite type condition, the author proved in [12] that for a class of intervals $J, \mu(J)$ are controlled by products of a family of non-negative matrices (see Proposition 5.1 and $\S 6$ for details). Applying this result and Theorem 1.1, we will prove the following general result:

Theorem 1.2. Let $\mu$ be the self-similar measure on $\mathbb{R}$ satisfying

$$
\mu=\sum_{j=1}^{\ell} p_{j} \mu \circ S_{j}^{-1},
$$

where $S_{j} x=\rho x+b_{j}, p_{j}>0$ and $\sum_{j=1}^{\ell} p_{j}=1$. Assume that $\left\{S_{j}\right\}_{j=1}^{\ell}$ satisfies the finite type condition. Then we can construct a family (finite or countably infinite) of closed intervals $I_{j}$ with disjoint interiors, such that $\mu$ is supported on $\bigcup_{j} I_{j}$ and the restricted measure $\left.\mu\right|_{I_{j}}$ of $\mu$ on each interval $I_{j}$ satisfies the complete multifractal formalism. Moreover the dimension spectrum $\operatorname{dim}_{H} E_{\left.\mu\right|_{I_{j}}}(\alpha)$ is independent of $j$.

For the global property of $\mu$, we remark that under the condition of Theorem 1.2, we do have $\operatorname{dim}_{H} E_{\mu}(\alpha)=\inf _{q \in \mathbb{R}}\{\alpha q-\tau(q)\}$ for all $\alpha \in\left[\tau^{\prime}(+\infty), \tau^{\prime}(0-)\right]$, where $\tau^{\prime}(+\infty):=\lim _{q \rightarrow+\infty} \tau^{\prime}(q)$. This extends slightly the aforementioned result in $[12,31]$ (which says the formula holds for all $\alpha=\tau^{\prime}(t)$ with $t>0$ ). Furthermore if the equality $\tau\left(\mu_{k}, q\right)=\tau(q)$ holds for all $q<0$ (for instance, this holds for the Erdös measure), then the complete multifractal formalism holds for $\mu$. For details, see Remark 5.3. 


\section{SOME BASIC LEMMAS}

Let $M(x)$ be a non-negative matrix-valued function defined on $\Sigma=\{1, \ldots, m\}^{\mathbb{N}}$ satisfying the assumptions (H1) and (H2). For $n \in \mathbb{N}$, let $\Sigma_{n}$ denote the set of all words of length $n$ over $\{1, \ldots, m\}$. Denote $\Sigma^{*}=\bigcup_{n=1}^{\infty} \Sigma_{n}$.

Lemma 2.1. There exists a constant $C>0$ such that

(i) for any $I \in \Sigma_{n}, J \in \Sigma_{\ell}$, there exists $K \in \bigcup_{k=1}^{r} \Sigma_{k}$ such that $\left\|M_{I K J}\right\| \geq C\left\|M_{I}\right\|\left\|M_{J}\right\|$.

(ii) for any $I \in \Sigma_{n}$ and $\ell \in \mathbb{N}$, there exists $K \in \Sigma_{\ell}$ such that $\left\|M_{I K}\right\| \geq C^{\ell}\left\|M_{I}\right\|$.

(iii) for any $I \in \Sigma_{n}, J \in \Sigma_{\ell}$, there exist $1 \leq k \leq r, K_{1} \in \Sigma_{k}$ and $K_{2} \in \Sigma_{2 r-k}$ such that $\left\|M_{I K_{1} J K_{2}}\right\| \geq C\left\|M_{I}\right\|\left\|M_{J}\right\|$.

(iv) for any $I_{1}, I_{2}, \ldots, I_{n} \in \Sigma_{\ell}$, there exist $K_{1}, K_{2}, \ldots, K_{2 n} \in \bigcup_{j=1}^{2 r-1} \Sigma_{j}$ such that $\sum_{j=1}^{2 n}\left|K_{j}\right|=2 n r$ and

$$
C^{n} \prod_{j=1}^{n}\left\|M_{I_{j}}\right\| \leq\left\|M_{I_{1}\left(K_{1} I_{2} K_{2}\right)\left(K_{3} I_{3} K_{4}\right) \ldots\left(K_{2 n-3} I_{n} K_{2 n-2}\right) K_{2 n-1} K_{2 n}}\right\| \leq C^{-n} \prod_{j=1}^{n}\left\|M_{I_{j}}\right\| .
$$

Proof. Assume $I \in \Sigma_{n}, J \in \Sigma_{\ell}$. Since $\sum_{k=1}^{r} H^{k}>\mathbf{0}$, we have

$$
\begin{aligned}
\sum_{k=1}^{r} \sum_{K \in \Sigma_{k}}\left\|M_{I K J}\right\| & =\left\|M_{I}\left(\sum_{k=1}^{r} H^{k}\right) M_{J}\right\| \\
& \geq D\left\|M_{I}\right\|\left\|M_{J}\right\|
\end{aligned}
$$

for some constant $D>0$. It follows that there exists $K \in \bigcup_{k=1}^{r} \Sigma_{k}$ such that

$$
\left\|M_{I K J}\right\| \geq \frac{D}{\#\left\{\bigcup_{k=1}^{r} \Sigma_{k}\right\}}\left\|M_{I}\right\|\left\|M_{J}\right\|
$$

where \# denotes the cardinality. This finishes the proof of part (i).

To prove part (ii) we can assume $M_{I} \neq \mathbf{0}$. By induction it suffices to prove part (ii) in the case $\ell=1$. Choose $j \in\{1, \ldots, m\}$ so that $M_{j} \neq \mathbf{0}$. By (i), there exist $1 \leq k \leq r$ and $i_{1} i_{2} \ldots i_{k} \in \Sigma_{k}$ such that

$$
\left\|M_{I i_{1} i_{2} \ldots i_{k} j}\right\| \geq C_{1}\left\|M_{I}\right\|\left\|M_{j}\right\|>0
$$

for some constant $C_{1}>0$. Since $\left\|M_{I i_{1}}\right\|\left\|M_{i_{2} \ldots i_{k} j}\right\| \geq\left\|M_{I i_{1} i_{2} \ldots i_{k} j}\right\|$, we have

$$
\left\|M_{I i_{1}}\right\| \geq \frac{C_{1}\left\|M_{j}\right\|}{\left\|M_{i_{2} \ldots i_{k} j}\right\|}\left\|M_{I}\right\| .
$$

This completes the proof of part (ii).

Part (iii) follows immediately from part (i) and part (ii).

To see part (iv), using part (iii) inductively, we may find $K_{1}, \ldots, K_{2 n-2}$ with $\left|K_{2 j-1}\right|+\left|K_{2 j}\right|=2 r$ for $1 \leq j \leq n-1$, such that

$$
\left\|M_{I_{1}\left(K_{1} I_{2} K_{2}\right)\left(K_{3} I_{3} K_{4}\right) \ldots\left(K_{2 n-3} I_{n} K_{2 n-2}\right)}\right\| \geq C^{n-1} \prod_{j=1}^{n}\left\|M_{I_{j}}\right\|
$$


Using part (ii) we may find $K_{2 n-1} K_{2 n} \in \Sigma_{2 r}$ such that the first inequality of (2.1) holds. Note that the second inequality also holds since

$$
\begin{aligned}
\left\|M_{I_{1}\left(K_{1} I_{2} K_{2}\right) \ldots\left(K_{2 n-3} I_{n} K_{2 n-2}\right) K_{2 n-1} K_{2 n}}\right\| & \leq\left(\prod_{j=1}^{n}\left\|M_{I_{j}}\right\|\right)\left(\prod_{j=1}^{2 n}\left\|M_{K_{j}}\right\|\right) \\
& \leq\left(\prod_{j=1}^{n}\left\|M_{I_{j}}\right\|\right)\left(\max _{1 \leq i \leq m}\left\|M_{i}\right\|\right)^{2 n r} .
\end{aligned}
$$

This finishes the proof of the lemma.

Lemma 2.2. The limit in defining $P_{M}(q)$ in (1.5) exists for any $q \in \mathbb{R}$. Moreover, $P_{M}(q)$ is a convex function.

Proof. Here we adopt a proof from [14]. Denote by $s_{n}=\sum_{J \in \mathcal{N}_{n}}\left\|M_{J}\right\|^{q}$.

We first consider the case $q \geq 0$. Since $\left\|M_{I J}\right\| \leq\left\|M_{I}\right\|\left\|M_{J}\right\|$ for any $I \in \mathcal{N}_{n}$ and $J \in \mathcal{N}_{\ell}$, the sequence $\left\{s_{n}\right\}$ is sub-multiplicative. Thus the $\operatorname{limit}_{n \rightarrow \infty} \frac{\log s_{n}}{n}$ exists.

Now assume $q<0$. By part (i) of Lemma 2.1, there is a constant $C>0$ such that for any $I \in \mathcal{N}_{n}$ and $J \in \mathcal{N}_{\ell}$, there exists $K \in \bigcup_{k=1}^{r} \Sigma_{k}$ satisfying

$$
0<\left\|M_{I K J}\right\| \leq C\left\|M_{I}\right\|\left\|M_{J}\right\| .
$$

It implies $s_{n} s_{\ell} \leq C^{-q} \sum_{k=1}^{r} s_{n+\ell+k}$. From (2.2) we also deduce that for any $I \in \Sigma^{*}$, there exists $i \in \Sigma_{1}$ such that $M_{I i} \neq 0$; Since $\left\|M_{I i}\right\| \leq C_{1}\left\|M_{I}\right\|$ for some constant $C_{1}>0$, we have $s_{n} \leq C_{1}^{-q} s_{n+1}$ for any integers $n, \ell$. It follows that $s_{n} s_{\ell} \leq C^{\prime} s_{n+\ell+r}$ for some constant $C^{\prime}>0$ (depending on $q$ ), which implies that $a_{n}=\frac{1}{C^{\prime}} s_{n-r}$ is super-multiplicative in the sense that $a_{n+\ell} \geq a_{n} a_{\ell}$ for all $n, \ell>r$. Hence the $\operatorname{limit}_{\lim } \rightarrow \infty \frac{\log s_{n}}{n}$ exists.

The convexity of $P_{M}(q)$ follows by a standard argument.

Let $L_{M}$ and $E_{M}(\cdot)$ be defined as in (1.2) and (1.3) respectively. It is clear that $L_{M}=\{\alpha \in \mathbb{R}$ : $\left.E_{M}(\alpha) \neq \emptyset\right\}$. Define

$$
\alpha_{M}=\lim _{n \rightarrow \infty} \frac{1}{n} \min _{I \in \mathcal{N}_{n}} \log \left\|M_{I}\right\|, \quad \beta_{M}=\lim _{n \rightarrow \infty} \frac{1}{n} \max _{I \in \mathcal{N}_{n}} \log \left\|M_{I}\right\| .
$$

Lemma 2.3. (i) The above limits in defining $\alpha_{M}$ and $\beta_{M}$ exist. Moreover, $\alpha_{M}, \beta_{M} \in \mathbb{R}$.

(ii) $L_{M}=\left[\alpha_{M}, \beta_{M}\right]$.

(iii) $\alpha_{M}=\lim _{q \rightarrow-\infty} \frac{P_{M}(q)}{q}$ and $\beta_{M}=\lim _{q \rightarrow+\infty} \frac{P_{M}(q)}{q}$.

Proof. Denote $u_{n}=\max _{I \in \mathcal{N}_{n}}\left\|M_{I}\right\|$ and $v_{n}=\min _{I \in \mathcal{N}_{n}}\left\|M_{I}\right\|$ for any $n \in \mathbb{N}$. It is clear that the sequence $\left\{u_{n}\right\}$ is sub-multiplicative. Thus the limit in defining $\beta_{M}$ exists. On the other hand by (2.2), there is a constant $C>0$ such that for any $n, m \in \mathbb{N}$, there exists $k$ between 1 and $r$ such that

$$
v_{n+m+k} \leq C v_{n} v_{m}
$$

Using (2.2) again we deduce that for any $I \in \mathcal{N}_{n}$ there exists $i \in \Sigma_{1}$ such that $\left\|M_{I i}\right\| \neq 0$. Since $\left\|M_{I i}\right\| \leq C_{1}\left\|M_{I}\right\|$ for some constants $C_{1}>0$, we have $v_{n+1} \leq C_{1} v_{n}$. This fact together with (2.4) yields $v_{n+m+r} \leq C^{\prime} v_{n} v_{m}$ for some constants $C^{\prime}>0$. It implies that the sequence $\left\{C^{\prime} v_{n-r}\right\}_{n}$ is sub-multiplicative. Thus the limit in defining $\alpha_{M}$ exists. To see $\alpha_{M}, \beta_{M} \in \mathbb{R}$, we only need to observe that

$$
t^{n} \leq v_{n} \leq u_{n} \leq\left(\max _{1 \leq i \leq m}\left\|M_{i}\right\|\right)^{n}
$$


where $t$ is the smallest non-zero entry appearing in the matrices $M_{i}, 1 \leq i \leq m$. This finishes the proof of part (i).

To see (ii), by the definitions of $L_{M}, \alpha_{M}$ and $\beta_{M}$ we have $L_{M} \subseteq\left[\alpha_{M}, \beta_{M}\right]$ immediately. Thus to complete the proof of part (ii), we only need to show that for any $s \in\left[\alpha_{M}, \beta_{M}\right]$, there exists $y \in \Sigma$ such that $\lambda_{M}(y)=s$. Now fix $s \in\left[\alpha_{M}, \beta_{M}\right]$ and write $s=p \alpha_{M}+(1-p) \beta_{M}$ for some $p \in[0,1]$. For $n \in \mathbb{N}$, construct a sequence of words $\left\{\omega_{n}\right\}_{n=1}^{\infty}$ such that $\omega_{n} \in \mathcal{N}_{n}$ and

$$
\left\|M_{\omega_{2 n}}\right\|=u_{2 n}, \quad\left\|M_{\omega_{2 n-1}}\right\|=v_{2 n-1} .
$$

Construct a sequence of positive integers $\left\{N_{n}\right\}$ by

$$
N_{n}= \begin{cases}\llbracket p n+\log n \rrbracket, & \text { if } n \text { is odd, } \\ \llbracket(1-p) n+\log n \rrbracket, & \text { otherwise, }\end{cases}
$$

where $\llbracket x \rrbracket$ denotes the integral part of $x$. It can be checked directly that

$$
\lim _{n \rightarrow \infty} N_{n}=\infty, \quad \lim _{n \rightarrow \infty} \frac{n N_{n}}{\sum_{i=1}^{n} i N_{i}}=0, \quad \lim _{n \rightarrow \infty} \frac{\sum_{i=1}^{n}(2 i-1) N_{2 i-1}}{\sum_{j=1}^{2 n} j N_{j}}=p .
$$

Now define a sequence of words in the following way:

$$
\underbrace{\omega_{1}, \ldots, \omega_{1}}_{N_{1}}, \underbrace{\omega_{2}, \ldots, \omega_{2}}_{N_{2}}, \ldots, \underbrace{\omega_{n}, \ldots, \omega_{n}}_{N_{n}}, \ldots
$$

We label this sequence as $\left\{\nu_{n}\right\}$. Using Lemma 2.1 (i) inductively, we can find a sequence of words $\left\{K_{n}\right\}$ such that $K_{n} \in \bigcup_{k=1}^{r} \Sigma_{k}$ and

$$
\left\|M_{\nu_{1} K_{1} \nu_{2} K_{2} \ldots \nu_{\ell} K_{\ell}}\right\| \geq C^{\ell} \prod_{k=1}^{\ell}\left\|M_{\nu_{k}}\right\|, \quad \forall \ell \in \mathbb{N},
$$

where $C$ is the constant in Lemma 2.1. Note that the left-hand side of the above inequality is not greater than $D^{r \ell} \prod_{k=1}^{\ell}\left\|M_{\nu_{k}}\right\|$, where $D=\max \left\{1, \max _{1 \leq i \leq m}\left\|M_{i}\right\|\right\}$. Define $y \in \Sigma$ by

$$
y=\nu_{1} K_{1} \nu_{2} K_{2} \ldots \nu_{\ell} K_{\ell} \ldots
$$

One can check directly that $\lambda_{M}(y)=s$ by using (2.5) and the above two inequalities about $\left\|M_{\nu_{1} K_{1} \nu_{2} K_{2} \ldots \nu_{\ell} K_{\ell}}\right\|$. This finishes the proof of (ii).

To see (iii), note that for each $n \in \mathbb{N}$,

$$
\begin{cases}\left(u_{n}\right)^{q} \leq \sum_{I \in \mathcal{N}_{n}}\left\|M_{I}\right\|^{q} \leq m^{n}\left(u_{n}\right)^{q}, & \forall q \geq 0 \\ \left(v_{n}\right)^{q} \leq \sum_{I \in \mathcal{N}_{n}}\left\|M_{I}\right\|^{q} \leq m^{n}\left(v_{n}\right)^{q}, & \forall q<0\end{cases}
$$

which implies that

$$
\begin{cases}q \beta_{M} \leq P_{M}(q) \leq \log m+q \beta_{M}, & \forall q \geq 0 \\ q \alpha_{M} \leq P_{M}(q) \leq \log m+q \alpha_{M}, & \forall q<0\end{cases}
$$

By taking the limits we obtain the desired result.

For any $\alpha \in \mathbb{R}, n \in \mathbb{N}$ and $\epsilon>0$, define

$$
F(\alpha ; n, \epsilon)=\left\{I \in \Sigma_{n}:\left|\frac{1}{n} \log \left\|M_{I}\right\|-\alpha\right| \leq \epsilon\right\}
$$

and $f(\alpha ; n, \epsilon)=\# F(\alpha ; n, \epsilon)$. Moreover for $\ell \in \mathbb{N}$, define

$$
F_{\ell}(\alpha ; n \ell, \epsilon)=\left\{i_{1} \ldots i_{n \ell} \in \Sigma_{n \ell}:\left|\frac{1}{n \ell} \log \prod_{j=1}^{n}\left\|M_{i_{(j-1) \ell+1} i_{(j-1) \ell+2} \ldots i_{j \ell}}\right\|-\alpha\right| \leq \epsilon\right\}
$$


and $f_{\ell}(\alpha ; n \ell, \epsilon)=\# F_{\ell}(\alpha ; n \ell, \epsilon)$.

Lemma 2.4. For any $\alpha \in \mathbb{R}, \epsilon>0$, there exists $\delta>0$ such that if $n, \ell \in \mathbb{N}$ satisfy $\ell / n<\delta$, then $f(\alpha ; n+\ell, 2 \epsilon) \geq f(\alpha ; n, \epsilon)$.

Proof. By Lemma 2.1, there is a constant $C>0$ such that for any $I \in F(\alpha ; n, \epsilon)$ and $\ell \in \mathbb{N}$, there exists $K \in \Sigma_{\ell}$ satisfying $\left\|M_{I K}\right\| \geq C^{\ell}\left\|M_{I}\right\|$. Meanwhile it is obvious that $\left\|M_{I K}\right\| \leq C_{1}^{\ell}\left\|M_{I}\right\|$, where $C_{1}=\max _{1 \leq i \leq m}\left\|M_{i}\right\|$. By these two inequalities, we have

$$
\frac{1}{n+\ell}\left(\ell \log C+\log \left\|M_{I}\right\|\right) \leq \frac{1}{n+\ell} \log \left\|M_{I K}\right\| \leq \frac{1}{n+\ell}\left(\ell \log C_{1}+\log \left\|M_{I}\right\|\right) .
$$

Thus there exists $\delta>0$ such that if $\ell / n<\delta$ then

$$
\left|\frac{\log \left\|M_{I K}\right\|}{n+\ell}-\alpha\right|<2 \epsilon .
$$

This implies $f(\alpha ; n+\ell, 2 \epsilon) \geq f(\alpha ; n, \epsilon)$.

Lemma 2.5. There is a constant $D>0$ such that for any $\epsilon>0$, there exists $N>0$ (depending on $\epsilon)$ such that for any $\alpha \in L_{M}$,

$$
f(\alpha ; p(\ell+2 r), 2 \epsilon) \geq D^{p} f_{\ell}(\alpha ; p \ell, \epsilon) \geq D^{p}(f(\alpha ; \ell, \epsilon))^{p}, \quad \forall p \in \mathbb{N}, \forall \ell>N .
$$

Proof. It is obvious that for any $I_{1}, \ldots, I_{p} \in F(\alpha ; \ell, \epsilon), I_{1} \ldots I_{p} \in F_{\ell}(\alpha ; p \ell, \epsilon)$. It implies that $f_{\ell}(\alpha ; p \ell ; \epsilon) \geq(f(\alpha ; \ell ; \epsilon))^{p}$. Thus the second inequality in (2.7) always holds.

To prove the first inequality, let $I_{1}, \ldots, I_{p} \in \Sigma_{\ell}$ be any words with $I_{1} \ldots I_{p} \in F_{\ell}(\alpha ; p \ell, \epsilon)$. By part (iv) of Lemma 2.1, there exist $K_{1}, \ldots, K_{2 p} \in \bigcup_{j=1}^{2 r-1} \Sigma_{j}$ such that $\sum_{j=1}^{2 p}\left|K_{j}\right|=2 p r$ and

$$
C^{p} \prod_{j=1}^{p}\left\|M_{I_{j}}\right\| \leq\left\|M_{I_{1}\left(K_{1} I_{2} K_{2}\right)\left(K_{3} I_{3} K_{4}\right) \ldots\left(K_{2 p-3} I_{p} K_{2 p-2}\right) K_{2 p-1} K_{2 p}}\right\| \leq C^{-p} \prod_{j=1}^{p}\left\|M_{I_{j}}\right\|,
$$

where $C$ is a constant. Hence if $\ell>\epsilon^{-1}\left(|\log C|+2 r \max \left\{|\beta|: \beta \in L_{M}\right\}\right)$, then

$$
I_{1}\left(K_{1} I_{2} K_{2}\right)\left(K_{3} I_{3} K_{4}\right) \ldots\left(K_{2 p-3} I_{p} K_{2 p-2}\right) K_{2 p-1} K_{2 p} \in F(\alpha ; p(\ell+2 r), 2 \epsilon) .
$$

Note that when $I_{1} \ldots I_{p}$ is taken over the set $F_{\ell}(\alpha ; p \ell, \epsilon)$, by the pigeon hole principle, we get at least

$$
\left(\#\left(\bigcup_{j=1}^{2 r-1} \Sigma_{j}\right)\right)^{-2 p} f_{\ell}(\alpha ; p \ell, \epsilon)
$$

many different elements of form $I_{1}\left(K_{1} I_{2} K_{2}\right)\left(K_{3} I_{3} K_{4}\right) \ldots\left(K_{2 p-3} I_{p} K_{2 p-2}\right) K_{2 p-1} K_{2 p}$ which belong to $F(\alpha ; p(\ell+2 r), 2 \epsilon)$. Here $\left(\#\left(\bigcup_{j=1}^{2 r-1} \Sigma_{j}\right)\right)^{2 p}$ is an upper bound of the number of all possible different elements $\left(K_{1}, \ldots, K_{2 p}\right)$. Taking $D=\left(\#\left(\bigcup_{j=1}^{2 r-1} \Sigma_{j}\right)\right)^{-2}$, we have $f(\alpha ; p(\ell+2 r), 2 \epsilon) \geq D^{p} f_{\ell}(\alpha ; p \ell, \epsilon)$. This finishes the proof of the lemma.

Fix $\ell \in \mathbb{N}$. Define

$$
E_{M}^{(\ell)}(\alpha)=\left\{\left(x_{i}\right)_{i=1}^{\infty} \in \Sigma: \lim _{n \rightarrow \infty} \frac{1}{n \ell} \log \left(\prod_{j=1}^{n}\left\|M_{x_{(j-1) \ell+1} \cdots x_{j \ell}}\right\|\right)=\alpha\right\}, \quad \alpha \in \mathbb{R}
$$

and

$$
P_{M}(\ell, q)=\frac{1}{\ell} \log \left(\Sigma_{J \in \mathcal{N}_{\ell}}\left\|M_{J}\right\|^{q}\right), \quad q \in \mathbb{R} .
$$


Proposition 2.6. If $\alpha=P_{M}^{\prime}(\ell, q)$ for some $q \in \mathbb{R}$, then $E_{M}^{(\ell)}(\alpha) \neq \emptyset$ and

$$
\operatorname{dim}_{H} E_{M}^{(\ell)}(\alpha)=\frac{1}{\log m}\left(P_{M}(\ell, q)-\alpha q\right) .
$$

The proof of the above result is based on the following theorem of Billingsley:

Proposition 2.7. (c.f. [19], P. 99, see also [5, 50]). Let $(X, d)$ be a metric space $\left(\mathbb{R}^{n}\right.$ or $\left.\Sigma\right)$. Let $\mu$ be a Borel probability measure on $X$. For a Borel set $E \subset X$, we have $a \leq \operatorname{dim}_{H} E \leq b$ if

$$
\mu(E)>0, \quad E \subset\left\{x \in X: a \leq \liminf _{r \rightarrow 0} \frac{\log \mu\left(B_{r}(x)\right)}{\log r} \leq b\right\} .
$$

Proof of Proposition 2.6. Assume $\alpha=P_{M}^{\prime}(\ell, q)$ for some $q \in \mathbb{R}$. Then

$$
\alpha=\frac{1}{\ell} e^{-\ell P_{M}(\ell, q)} \sum_{J \in \mathcal{N}_{\ell}}\left\|M_{J}\right\|^{q} \log \left\|M_{J}\right\| .
$$

Define a product-like measure $\mu$ on $\Sigma$ such that

$$
\mu\left(\left[x_{1} x_{2} \ldots x_{n \ell}\right]\right)=e^{-n \ell P_{M}(\ell, q)} \prod_{j=1}^{n}\left\|M_{x_{(j-1) \ell+1} \cdots x_{j \ell}}\right\|^{q}
$$

for any $n$ and any cylinder $\left[x_{1} x_{2} \ldots x_{n \ell}\right]$ with $x_{(j-1) \ell+1} \cdots x_{j \ell} \in \mathcal{N}_{\ell}$ for each $1 \leq j \leq n$. It is clear $\mu$ is $\sigma^{\ell}$-invariant and $\sigma^{\ell}$-ergodic. By the Birkhoff's ergodic theorem, we have for $\mu$ a.e. $x=\left(x_{i}\right)_{i=1}^{\infty} \in \Sigma$,

Note also that

$$
\begin{aligned}
\lim _{n \rightarrow \infty} \frac{\log \mu\left(\left[x_{1} x_{2} \ldots x_{n \ell}\right]\right)}{\log m^{-n \ell}} & =\lim _{n \rightarrow \infty} \frac{-n \ell P_{M}(\ell, q)+\sum_{j=1}^{n} q \log \left\|M_{x_{(j-1) \ell+1} \cdots x_{j}}\right\|}{-n \ell \log m} \\
& =\frac{1}{\log m}\left(P_{M}(\ell, q)-q \ell^{-1} e^{-\ell P_{M}(\ell, q)} \sum_{J \in \mathcal{N}_{\ell}}\left\|M_{J}\right\|^{q} \log \left\|M_{J}\right\|\right) \\
& =\frac{1}{\log m}\left(P_{M}(\ell, q)-\alpha q\right) .
\end{aligned}
$$

$$
\left(x_{i}\right)_{i=1}^{\infty} \in E_{M}^{(\ell)}(\alpha) \Longleftrightarrow \lim _{n \rightarrow \infty} \frac{\log \mu\left(\left[x_{1} x_{2} \ldots x_{n \ell}\right]\right)}{\log m^{-n \ell}}=\frac{1}{\log m}\left(P_{M}(\ell, q)-\alpha q\right) .
$$

This proves the proposition by using the Billingsley theorem.

Proposition 2.8. Let $\left\{M_{i}: i=1, \ldots, m\right\}$ be a family of $d \times d$ complex matrices which is irreducible in the following sense: there is no proper linear subspace $V$ of $\mathbb{C}^{d}$ such that $M_{i} V \subseteq V$ for all $1 \leq i \leq m$. Then there exist $C>0$ and $k \in \mathbb{N}$ such that for any words $I, J \in \Sigma^{*}=\bigcup_{n=1}^{\infty}\{1, \ldots, m\}^{n}$, there exists a word $K \in \bigcup_{n=1}^{k}\{1, \ldots, m\}^{n}$ such that

$$
\left\|M_{I K J}\right\| \geq C\left\|M_{I}\right\|\left\|M_{J}\right\|,
$$

where $\|\cdot\|$ denotes the standard matrix norm.

Proof. We prove the proposition by contradiction. Assume that the proposition is not true. Then for any $\delta>0$ and $n \in \mathbb{N}$, there exist $I, J \in \Sigma^{*}$ such that $M_{I}, M_{J} \neq \mathbf{0}$ and

$$
\left\|M_{I K J}\right\| \leq \delta\left\|M_{I}\right\|\left\|M_{J}\right\|, \quad \forall K \in \Sigma^{*} \text { with }|K| \leq n,
$$

where $|K|$ denotes the length of $K$. Let $u_{I}$ be a unit row vector and $v_{J}$ a unit column vector in $\mathbb{C}^{d}$ such that

$$
\left\|M_{I}\right\|=\left\|u_{I} M_{I}\right\|, \quad\left\|M_{J}\right\|=\left\|M_{J} v_{J}\right\| .
$$


Define $\widetilde{u_{I}}=\frac{u_{I} M_{I}}{\left\|M_{I}\right\|}$ and $\widetilde{v_{J}}=\frac{M_{J} v_{J}}{\left\|M_{J}\right\|}$. Then

$$
\left|\widetilde{u_{I}} M_{K} \widetilde{v_{J}}\right| \leq \delta, \quad \forall K \in \Sigma^{*} \text { with }|K| \leq n .
$$

Take two sequences $\left\{\delta_{\ell}\right\} \downarrow 0$ and $\left\{n_{\ell}\right\} \uparrow \infty$. Then for each $\ell$, there are unit column vectors $w_{\ell, 1}$ and $w_{\ell, 2}$ such that

$$
\left|w_{\ell, 1}^{t} M_{K} w_{\ell, 2}\right| \leq \delta_{\ell}, \quad \forall K \in \Sigma^{*} \text { with }|K| \leq n_{\ell} .
$$

Let $\left(w_{1}, w_{2}\right)$ be a limit point of $\left\{\left(w_{\ell, 1}, w_{\ell, 2}\right)\right\}_{\ell \in \mathbb{N}}$. Then we have

$$
\left|w_{1}^{t} M_{K} w_{2}\right|=0, \quad \forall K \in \Sigma^{*} .
$$

Let $V$ be the linear space spanned by $\left\{M_{K} w_{2}: K \in \Sigma^{*}\right\}$. It is clear that $M_{i} V \subseteq V$ for all $1 \leq i \leq m$. However $V$ is perpendicular to $w_{1}$, which means that $V$ is a proper subspace of $\mathbb{C}^{d}$. This contradicts the irreducibility of $\left\{M_{i}: 1 \leq i \leq m\right\}$.

We remark that the above result and its extension for singular value functions for matrix products were independently obtained by Falconer and Sloan [22]. They also obtained partial results on the multifractal analysis of norms of matrix products [23].

\section{A Dimension formula for $E_{M}(\alpha)$}

In this section we give a dimensional formula for the set $E_{M}(\alpha)$.

Proposition 3.1. For $\alpha \in L_{M}$, we have

$$
\lim _{\epsilon \rightarrow 0} \liminf _{n \rightarrow \infty} \frac{\log f(\alpha ; n, \epsilon)}{\log m^{n}}=\lim _{\epsilon \rightarrow 0} \limsup _{n \rightarrow \infty} \frac{\log f(\alpha ; n, \epsilon)}{\log m^{n}} \quad\left(=: \Lambda_{M}(\alpha)\right) .
$$

The function $\Lambda_{M}: L_{M} \rightarrow[0,1]$ is concave and continuous.

Proof. The proposition follows from Lemma 2.4 and Lemma 2.5 by a standard argument. For a similar approach, see the proof of Proposition 3.2 in [11].

Proposition 3.2. For $\alpha \in L_{M}$, we have

$$
\operatorname{dim}_{H} E_{M}(\alpha)=\operatorname{dim}_{P} E_{M}(\alpha)=\Lambda_{M}(\alpha)
$$

Furthermore, for any word $\xi \in \Sigma^{*}$ with $M_{\xi} \neq \mathbf{0}$, there exists $\Gamma=\Gamma(\xi) \subset E_{M}(\alpha)$ such that $\operatorname{dim}_{H} \Gamma=$ $\Lambda_{M}(\alpha)$ and for any $x=\left(x_{j}\right)_{j=1}^{\infty} \in \Gamma$, there exists a sequence of positive integers $\left\{n_{k}(x)\right\}_{k=1}^{\infty} \uparrow \infty$ such that $n_{1}(x)=1, \lim _{k \rightarrow \infty} \frac{n_{k+1}(x)}{n_{k}(x)}=1$, and $\xi$ appears in $\left(x_{j}\right)_{j=1}^{\infty}$ at each position $n_{k}(x)$ (here we say $\xi=u_{1} \ldots u_{s}$ appears in $\left(x_{j}\right)_{j=1}^{\infty}$ at position $n$ if $\left.x_{n} x_{n+1} \ldots x_{n+s-1}=\xi\right)$.

Proof. We divide the proof into two small steps.

Step 1. $\operatorname{dim}_{P} E_{M}(\alpha) \leq \Lambda_{M}(\alpha), \forall \alpha \in L_{M}$.

The proof of this step is standard. Let

$$
G(\alpha ; k, \epsilon)=\bigcap_{n=k}^{\infty}\left\{x=\left(x_{i}\right)_{i=1}^{\infty} \in \Sigma:\left|\frac{1}{n} \log \left\|M_{x_{1}} \cdots M_{x_{n}}\right\|-\alpha\right|<\epsilon\right\} .
$$

It is clear that for any $\epsilon>0$,

$$
E_{M}(\alpha) \subset \bigcup_{k=1}^{\infty} G(\alpha ; k, \epsilon)
$$


Notice that if $n \geq k, G(\alpha ; k, \epsilon)$ is covered by the union of all cylinders $[\omega]$ with $\omega \in F(\alpha ; n, \epsilon)$ whose total number is $f(\alpha ; n, \epsilon)$. Therefore we have the following estimate

$$
\overline{\operatorname{dim}}_{B} G(\alpha ; k, \epsilon) \leq \limsup _{n \rightarrow \infty} \frac{\log f(\alpha ; n, \epsilon)}{\log m^{n}} \quad(\forall \epsilon>0, \forall k \geq 1),
$$

where $\overline{\operatorname{dim}}_{B}$ denotes the upper box dimension (see [20]). On the other hand, by using the $\sigma$-stability of the packing dimension, we have

$$
\begin{aligned}
\operatorname{dim}_{P} E_{M}(\alpha) & \leq \operatorname{dim}_{P}\left(\bigcup_{k=1}^{\infty} G(\alpha ; k, \epsilon)\right) \leq \sup _{k} \operatorname{dim}_{P} G(\alpha ; k, \epsilon) \\
& \leq \sup _{k} \overline{\operatorname{dim}}_{B} G(\alpha ; k, \epsilon) \leq \limsup _{n \rightarrow \infty} \frac{\log f(\alpha ; n, \epsilon)}{\log m^{n}}
\end{aligned}
$$

Taking $\epsilon \rightarrow 0$, we obtain the desired result.

Step 2. For any given $\xi \in \Sigma^{*}$ with $M_{\xi} \neq \mathbf{0}$ and $\delta>0$, there exists $\Gamma \subset E_{M}(\alpha)$ such that $\operatorname{dim}_{H} \Gamma>\Lambda_{M}(\alpha)-\delta$ and for any $x=\left(x_{j}\right)_{j \geq 1} \in \Gamma$, there exists a sequence of positive integers $\left\{n_{k}(x)\right\}_{k=1}^{\infty} \uparrow \infty$ such that $n_{1}(x)=1, \lim _{k \rightarrow \infty} \frac{n_{k+1}(x)}{n_{k}(x)}=1$ and $\xi$ appears in $\left(x_{j}\right)_{j \geq 1}$ at each position $n_{k}(x)$.

We first construct for any index $(i, j) \in\{1,2, \ldots, d\}^{2}$, a word $\eta(i, j) \in \Sigma^{*}$ such that $\xi$ appears in $\eta(i, j)$ as a subword and the $(i, j)$-th entry of $M_{\eta(i, j)}$ is positive. To achieve this purpose, we fix an index $(s, t)$ such that the $(s, t)$-th entry of $M_{\xi}$ is positive. By the assumption (H2), for any $1 \leq i, j \leq d$, there exist words $I_{1}, I_{2} \in \Sigma^{*}$ such that the $(i, s)$-th entry of $M_{I_{1}}$ and the $(t, j)$-th entry of $M_{I_{2}}$ are positive. Set $\eta(i, j)=I_{1} \xi I_{2}$. Then $\eta(i, j)$ satisfies the desired property.

By Proposition 3.1, there are $\ell_{j} \uparrow \infty$ and $\epsilon_{j} \downarrow 0$ such that

$$
f\left(\alpha ; \ell_{j}, \epsilon_{j}\right)>m^{\ell_{j}\left(\Lambda_{M}(\alpha)-\frac{\delta}{2}\right)} .
$$

Define a new sequence $\left\{\ell_{j}^{*}\right\}$ in the following manner

$$
\underbrace{\ell_{1}, \ldots, \ell_{1}}_{N_{1}} ; \underbrace{\ell_{2}, \ldots, \ell_{2}}_{N_{2}} ; \ldots ; \underbrace{\ell_{j}, \ldots, \ell_{j}}_{N_{j}} ; \ldots
$$

where $N_{j}$ is defined recursively by

$$
N_{j}=2^{\ell_{j+1}+N_{j-1}} \quad(j \geq 2) ; \quad N_{1}=1 .
$$

Set $\epsilon_{j}^{*}=\epsilon_{i}$ when $\ell_{j}^{*}=\ell_{i}$. By the pigeon hole principle, for each $j$, there exist an index $\left(s_{j}, t_{j}\right)$ and a subclass $G_{j}$ of $F\left(\alpha ; \ell_{j}^{*}, \epsilon_{j}^{*}\right)$ such that $\# G_{j} \geq d^{-2} f\left(\alpha ; \ell_{j}^{*}, \epsilon_{j}^{*}\right)$ and for any $I \in G_{j}$, the $\left(s_{j}, t_{j}\right)$-th entry of $M_{I}$ is greater than or equal to $d^{-2}\left\|M_{I}\right\|$.

Define

$$
\Theta^{*}=\prod_{j=1}^{\infty} G_{j}
$$

Observe that $\Theta^{*}$ is a homogeneous Moran set in $\Sigma$ (see, e.g., [11]). More precisely $\Theta^{*}$ is constructed as follows. At level 0 , we have only the initial cylinder $\Sigma$. In step $j$, cut a cylinder of level $j-1$ into 
$m^{\ell_{j}^{*}}$ cylinders and pick up $\# G_{j}$ ones. Thus we have (see, e.g., [11, Proposition 3.1])

$$
\begin{aligned}
\operatorname{dim}_{H} \Theta^{*} & \geq \liminf _{k \rightarrow \infty} \frac{\log \prod_{j=1}^{k} \# G_{j}}{\log \prod_{j=1}^{k+1} m^{\ell_{j}^{*}}} \\
& \geq \liminf _{k \rightarrow \infty} \frac{\log \prod_{j=1}^{k}\left(d^{-2} f\left(\alpha ; \ell_{j}^{*}, \epsilon_{j}^{*}\right)\right)}{\log \prod_{j=1}^{k+1} m^{\ell_{j}^{*}}} \\
& \geq \Lambda_{M}(\alpha)-\delta .
\end{aligned}
$$

Now for any $y^{*}=I_{1} I_{2} \ldots \in \Theta^{*}$ with $I_{j} \in G_{j}$, we define

$$
y^{* *}=\xi \eta\left(t, s_{1}\right) I_{1} \eta\left(t_{1}, s_{2}\right) I_{2} \eta\left(t_{2}, s_{3}\right) \ldots I_{j} \eta\left(t_{j}, s_{j+1}\right) \ldots
$$

Define $\Theta^{* *} \subset \Sigma$ as the set of sequences $y^{* *}$. By considering the map $y^{*} \mapsto y^{* *}$ which is nearly bi-Lipschitz, we have $\operatorname{dim}_{H} \Theta^{* *}=\operatorname{dim}_{H} \Theta^{*}$. On the other hand a direct check shows $\Theta^{* *} \subset E_{M}(\alpha)$. Set $\Gamma=\Theta^{* *}$. Then $\Gamma$ satisfies the desired property. This finishes the proof of the proposition.

\section{Proof of Theorem 1.1}

To prove Theorem 1.1, we need the following

Proposition 4.1. [11, Propostion 2.5]. Let $f$ be a convex real-valued function on $\mathbb{R}$. Denote

$$
a=\lim _{x \rightarrow-\infty} \frac{f(x)}{x}, \quad b=\lim _{x \rightarrow \infty} \frac{f(x)}{x} .
$$

(i) Suppose that $\left\{f_{n}\right\}_{n=1}^{\infty}$ is a sequence of differentiable convex functions converging to $f$ pointwise. Then for any $c \in(a, b)$, there exist $N>0$ and a uniformly bounded sequence of real numbers $\left\{x_{n}\right\}_{n \geq N}$ such that $f_{n}^{\prime}\left(x_{n}\right)=c$.

(ii) Assume $-\infty<a<b<\infty$. Then we have

$$
\varlimsup_{z \uparrow b} \inf _{x \in \mathbb{R}}\{-z x+f(x)\} \geq \inf _{x \in \mathbb{R}}\{-b x+f(x)\}, \quad \varlimsup_{z \downarrow a} \inf _{x \in \mathbb{R}}\{-z x+f(x)\} \geq \inf _{x \in \mathbb{R}}\{-a x+f(x)\} .
$$

Proof of Theorem 1.1. We divide the proof into 2 steps:

Step 1. $\operatorname{dim}_{P} E_{M}(\alpha) \leq \frac{1}{\log m}\left(-\alpha q+P_{M}(q)\right) \quad \forall \alpha \in L_{M}, \forall q \in \mathbb{R}$.

For any $\alpha \in L_{M}, \epsilon>0$ and $n \in \mathbb{N}$, let $f(\alpha ; n, \epsilon)$ be defined as in Section 2. Then

$$
\sum_{I \in \mathcal{N}_{n}}\left\|M_{I}\right\|^{q} \geq \begin{cases}f(\alpha ; n, \epsilon) \exp (n q(\alpha-\epsilon)), & \text { if } q \geq 0, \\ f(\alpha ; n, \epsilon) \exp (n q(\alpha+\epsilon)), & \text { if } q<0,\end{cases}
$$

which implies that for any $q \in \mathbb{R}$,

$$
P_{M}(q) \geq q \alpha+\lim _{\epsilon \rightarrow \infty} \liminf _{n \rightarrow \infty} \frac{\log f(\alpha ; n, \epsilon)}{n} .
$$

Applying Propositions 3.1 and 3.2 gives

$$
P_{M}(q) \geq \alpha q+(\log m) \operatorname{dim}_{P} E_{M}(\alpha) .
$$

Step 2. For all $\alpha \in L_{M}$,

$$
\operatorname{dim}_{H} E_{M}(\alpha) \geq \frac{1}{\log m} \inf _{q \in \mathbb{R}}\left\{-\alpha q+P_{M}(q)\right\} .
$$


To show the above inequality, we at first consider a trivial case: $\alpha_{M}=\beta_{M}$, where $\alpha_{M}$ and $\beta_{M}$ are defined as in (2.3). In this case, $\lambda_{M}(x)=\alpha_{M}$ for all $x \in \Sigma$. Thus by (2.6), we have

$$
\operatorname{dim}_{H} E_{M}\left(\alpha_{M}\right)=\operatorname{dim}_{H} \Sigma=1 \geq \frac{1}{\log m} \inf _{q \in \mathbb{R}}\left\{-\alpha_{M} q+P_{M}(q)\right\}
$$

In the following part we assume $\alpha_{M} \neq \beta_{M}$.

First we consider $\alpha \in\left(\alpha_{M}, \beta_{M}\right)$. The case $\alpha=\alpha_{M}$ or $\beta_{M}$ will be treated later. Let $P_{M}(\ell, q)$ and $E_{M}^{(\ell)}(\alpha)$ be defined as in $(2.9)$ and $(2.8)$ respectively. By Lemma 2.2, the sequence $\left\{P_{M}(\ell, q)\right\}_{\ell=1}^{\infty}$ converges to $P_{M}(q)$ pointwisely. Thus by Proposition 4.1, there exists $N>0$ such that there is a uniformly bounded sequence of real number $\left\{q_{\ell}\right\}_{\ell \geq N}$ such that $P_{M}^{\prime}\left(\ell, q_{\ell}\right)=\alpha$. By Proposition 2.6,

$$
\operatorname{dim}_{H} E_{M}^{(\ell)}(\alpha)=P_{M}\left(\ell, q_{\ell}\right)-\alpha q_{\ell}, \quad \forall \ell \geq N .
$$

Note that for any $\epsilon>0$ and $n \in \mathbb{N}$, the collection $\left\{[J]: J \in F_{\ell}(\alpha ; n \ell, \epsilon)\right\}$ is a $m^{-n \ell}$-cover of $E_{M}^{(\ell)}(\alpha)$. This implies

$$
\liminf _{n \rightarrow \infty} \frac{\log f_{\ell}(\alpha ; n \ell, \epsilon)}{n \ell \log m} \geq \operatorname{dim}_{H} E_{M}^{(\ell)}(\alpha)=P_{M}\left(\ell, q_{\ell}\right)-\alpha q_{\ell} .
$$

Combining it with $(2.7)$ yields

$$
\liminf _{n \rightarrow \infty} \frac{\log f(\alpha ; n \ell+2 n r, 2 \epsilon)}{(n \ell+2 n r) \log m} \geq \frac{\ell}{\ell+2 r} \cdot \frac{1}{\log m}\left(P_{M}\left(\ell, q_{\ell}\right)-\alpha q_{\ell}\right), \quad \forall \epsilon>0 .
$$

Hence by Proposition 3.2, we have

$$
\operatorname{dim}_{H} E_{M}(\alpha) \geq \frac{\ell}{\ell+2 r} \cdot \frac{1}{\log m}\left(P_{M}\left(\ell, q_{\ell}\right)-\alpha q_{\ell}\right), \quad \forall \ell \geq N
$$

Since the sequence $\left\{q_{\ell}\right\}_{\ell \geq N}$ is uniformly bounded, there exists a subsequence $\left\{q_{\ell_{i}}\right\}$ which converges to a finite point $q_{\infty}$. We are going to show

$$
\lim _{i \rightarrow \infty}\left(P_{M}\left(\ell_{i}, q_{\ell_{i}}\right)-\alpha q_{\ell_{i}}\right)=P_{M}\left(q_{\infty}\right)-\alpha q_{\infty} .
$$

Denote $t=\max \left\{\left\|M_{i}\right\|: 1 \leq i \leq m\right\}$, and define a new matrix function $\tilde{M}:=\frac{1}{t} M$. It is easily checked that

$$
P_{M}(\ell, q)=q \log t+P_{\tilde{M}}(\ell, q), \quad P_{M}(q)=q \log t+P_{\tilde{M}}(q) .
$$

Since $\left\|\tilde{M}_{i}\right\| \leq 1$ for all $1 \leq i \leq m, P_{\tilde{M}}(\ell, q)$ and $P_{\tilde{M}}(q)$ are monotone decreasing functions of $q$. Now for any $\epsilon>0$, we can find $a, b \in \mathbb{R}$ with $a<q_{\infty}<b$ such that

$$
P_{\tilde{M}}(a)<P_{\tilde{M}}\left(q_{\infty}\right)+\epsilon, \quad P_{\tilde{M}}(b)>P_{\tilde{M}}\left(q_{\infty}\right)-\epsilon .
$$

When $i$ is large enough, $q_{\ell_{i}} \in(a, b)$, and thus

$$
P_{\tilde{M}}\left(\ell_{i}, b\right) \leq P_{\tilde{M}}\left(\ell_{i}, q_{\ell_{i}}\right) \leq P_{\tilde{M}}\left(\ell_{i}, a\right) .
$$

It follows that

$$
\limsup _{i \rightarrow \infty} P_{\tilde{M}}\left(\ell_{i}, q_{\ell_{i}}\right) \leq \lim _{i \rightarrow \infty} P_{\tilde{M}}\left(\ell_{i}, a\right)=P_{\tilde{M}}(a)<P_{\tilde{M}}\left(q_{\infty}\right)+\epsilon
$$

and

$$
\liminf _{i \rightarrow \infty} P_{\tilde{M}}\left(\ell_{i}, q_{\ell_{i}}\right) \geq \lim _{i \rightarrow \infty} P_{\tilde{M}}\left(\ell_{i}, b\right)=P_{\tilde{M}}(b)>P_{\tilde{M}}\left(q_{\infty}\right)-\epsilon .
$$

Since $\epsilon>0$ is arbitrary, we have

$$
\lim _{i \rightarrow \infty} P_{\tilde{M}}\left(\ell_{i}, q_{\ell_{i}}\right)=P_{\tilde{M}}\left(q_{\infty}\right)
$$


Combining it with (4.5), we obtain $\lim _{i \rightarrow \infty} P_{M}\left(\ell_{i}, q_{\ell_{i}}\right)=P_{M}\left(q_{\infty}\right)$, which implies (4.4). Thus by (4.3), we have

This establishes (4.2).

$$
\operatorname{dim}_{H} E_{M}(\alpha) \geq \frac{1}{\log m}\left(P_{M}\left(q_{\infty}\right)-\alpha q_{\infty}\right)
$$

Now we consider the case $\alpha=\alpha_{M}$ (the case $\alpha=\beta_{M}$ can be treated in an identical way). By Proposition 3.2 and 3.1, the function $\operatorname{dim}_{H} E_{M}(x)$ of $x$ is continuous on $L_{M}$. Consequently,

and thus

$$
\operatorname{dim}_{H} E_{M}\left(\alpha_{M}\right)=\lim _{z \downarrow \alpha_{M}} \operatorname{dim}_{H} E_{M}(z),
$$

By Proposition 4.1 (ii), we have

$$
\operatorname{dim}_{H} E_{M}\left(\alpha_{M}\right) \geq \frac{1}{\log m} \lim _{z \downarrow \alpha_{M}} \inf _{q \in \mathbb{R}}\left\{-z q+P_{M}(q)\right\} .
$$

$$
\operatorname{dim}_{H} E_{M}\left(\alpha_{M}\right) \geq \frac{1}{\log m} \inf _{q \in \mathbb{R}}\left\{-\alpha_{M} q+P_{M}(q)\right\}
$$

which finishes the proof of (4.2).

\section{Proof of Theorem 1.2}

Let $S_{j} x=\rho x+b_{j}(j=1,2, \ldots, \ell)$ satisfy the finite type condition. That is, there exists a finite set $\Gamma$ of non-negative numbers such that for each integer $n \in \mathbb{N}$ and any two indices $J=j_{1} \ldots j_{n}$ and $J^{\prime}=j_{1}^{\prime} \ldots j_{n}^{\prime}$,

$$
\text { either } \quad \rho^{-n}\left|S_{J}(0)-S_{J^{\prime}}(0)\right|>c \quad \text { or } \quad \rho^{-n}\left|S_{J}(0)-S_{J^{\prime}}(0)\right| \in \Gamma,
$$

where $S_{J}$ denotes the composition $S_{j_{1}} \circ \ldots \circ S_{j_{n}}$ and $c=(1-\rho)^{-1}\left(\max _{1 \leq j \leq \ell} b_{j}-\min _{1 \leq i \leq \ell} b_{i}\right)$. Let $\mu$ be the self-similar measure generated by $\left\{S_{j}\right\}_{j=1}^{\ell}$ with the probability weight $\left\{p_{j}\right\}_{j=1}^{\ell^{\prime}}$, i.e., $\mu$ is the unique Borel probability measure on $\mathbb{R}$ satisfying the following relation:

$$
\mu=\sum_{j=1}^{\ell} p_{j} \mu \circ S_{j}^{-1}
$$

Let $K$ denote the self-similar set generated by $\left\{S_{j}\right\}_{j=1}^{\ell}$, i.e., $K$ is the unique non-empty compact set satisfying $K=\bigcup_{j=1}^{\ell} S_{j}(K)$.

The following proposition describes the local structure of $\mu$ :

Proposition 5.1. Let the family $\left\{S_{j}\right\}_{j=1}^{\ell}$ and the self-similar measure $\mu$ be given as above. Then there exist two positive integers $m$ and $d$, an $m \times m$ irreducible 0-1 matrix $A=\left(A_{i j}\right)_{1 \leq i, j \leq m}, m$ $d \times d$ non-negative matrices $M_{i}(1 \leq i \leq m)$, such that

(a) $\sum_{j=1}^{m} M_{j}$ is irreducible.

(b) $M_{j_{1}} M_{j_{2}} \ldots M_{j_{n}} \neq \mathbf{0}$ if and only if the word $J=j_{1} j_{2} \ldots j_{n}$ is A-admissible (i.e., $A_{j_{k} j_{k+1}}=1$ for $k=1, \ldots, n-1)$.

(c) Let $\Sigma_{A}$ denote the set $\left\{\left(x_{i}\right)_{i=1}^{\infty} \in \Sigma: A_{x_{i} x_{i+1}}=1\right.$ for all $\left.i \geq 1\right\}$. Then there exists a family (finite or countably infinite) of closed intervals $\left\{I_{k}\right\}_{k \in \Lambda}$ with disjoint interiors such that the endpoints of $I_{k}$ belong to $K$ for all $k \in \Lambda, \mu\left(\bigcup_{k \in \Lambda} I_{k}\right)=1$, and for each $k \in \Lambda$ there exists a surjective map $\pi_{k}: \Sigma_{A} \cap[1] \rightarrow K \cap I_{k}$ such that there are $C_{1}, C_{2}>1$ (only depending on $k)$, such that for any $n \in \mathbb{N}$ and $A$-admissible word $i_{1} i_{2} \ldots i_{n}$ with $i_{1}=1$, 
(c.1) $\frac{1}{C_{1}} \rho^{n} \leq \operatorname{diam} \pi_{k}\left(\Sigma_{A} \cap\left[i_{1} i_{2} \ldots i_{n}\right]\right) \leq C_{1} \rho^{n}$.

(c.2) $\frac{1}{C_{2}}\left\|M_{i_{1}} \ldots M_{i_{n}}\right\| \leq \mu\left(\pi_{k}\left(\Sigma_{A} \cap\left[i_{1} i_{2} \ldots i_{n}\right]\right)\right) \leq C_{2}\left\|M_{i_{1}} \ldots M_{i_{n}}\right\|$.

(c.3) The subintervals conv $\left(\pi_{k}\left(\Sigma_{A} \cap\left[i_{1} i_{2} \ldots i_{n} i_{n+1}\right]\right)\right.$ ) (where $i_{n+1}$ 's are taken so that $A_{i_{n} i_{n+1}}=$ 1) have disjoint interiors. Here conv $(Y)$ denotes the convex hull of $Y$. Moreover if we set $[a, b]:=\operatorname{conv}\left(\pi_{k}\left(\Sigma_{A} \cap\left[i_{1} i_{2} \ldots i_{n}\right]\right)\right)$ and $[c, d]:=\operatorname{conv}\left(\pi_{k}\left(\Sigma_{A} \cap\left[i_{1} i_{2} \ldots i_{n} i_{n+1}\right]\right)\right)$, then the ratios $\frac{d-c}{b-a}$ and $\frac{d-a}{b-a}$ only depend on $i_{n}$ and $i_{n+1}$.

Most parts of the above proposition were proved or implicit in [12]. However for the convenience of the readers and for completeness, we will provide a detailed constructive proof in the next section.

Proposition 5.2. Let $m, M_{i}(1 \leq i \leq m)$ and $\left\{I_{k}\right\}_{k \in \Lambda}$ be given as in the above proposition. Let $(\Sigma, \sigma)$ be the full shift space over the alphabet $\{1, \ldots, m\}$. For any $k \in \Lambda$ denote $\mu_{k}=\mu \mid I_{k}$, i.e. $\mu_{k}$ is the restriction of the measure $\mu$ on $I_{k}$. Then for any $q \in \mathbb{R}$,

$$
\tau\left(\mu_{k}, q\right)=P_{M}(q) / \log \rho, \quad \forall k \in \Lambda,
$$

where $P_{M}(q)$ is defined as in (1.5). Moreover $\tau\left(\mu_{k}, q\right)=\tau(\mu, q)$ for all $q \geq 0$.

Proof. The proof is identical to that of Proposition 5.7 and Lemma 5.3 in [12].

Proof of Theorem 1.2. We use the same notion as in Proposition 5.1. Fix one index $k \in \Lambda$ and consider the measure $\mu_{k}$. For $t \in \mathbb{R}$, define

$$
E_{t}=\left\{y \in \mathbb{R}: \lim _{\delta \rightarrow 0} \frac{\log \mu_{k}([y-\delta, y+\delta])}{\log \delta}=t\right\} .
$$

To prove Theorem 1.2, we shall prove the following two statements respectively:

(S1) $E_{t} \neq \emptyset \Longleftrightarrow t \in\left[\alpha_{\min }, \alpha_{\max }\right]$, where

and

$$
\alpha_{\min }:=\lim _{q \rightarrow+\infty} \frac{\tau\left(\mu_{k}, q\right)}{q}=\lim _{q \rightarrow+\infty} \frac{P_{M}(q)}{q \log \rho}
$$

(S2) For any $t \in\left[\alpha_{\min }, \alpha_{\max }\right]$,

$$
\alpha_{\max }:=\lim _{q \rightarrow-\infty} \frac{\tau\left(\mu_{k}, q\right)}{q}=\lim _{q \rightarrow-\infty} \frac{P_{M}(q)}{q \log \rho}
$$

$$
\operatorname{dim}_{H} E_{t}=\inf _{q \in \mathbb{R}}\left\{t q-\tau\left(\mu_{k}, q\right)\right\}=-\frac{1}{\log \rho} \inf _{q \in \mathbb{R}}\left\{-t q \log \rho+P_{M}(q)\right\} .
$$

By Lemma 2.3 and Proposition 5.2, we have

$$
\lim _{q \rightarrow+\infty} \frac{\tau\left(\mu_{k}, q\right)}{q}=\lim _{q \rightarrow+\infty} \frac{P_{M}(q)}{q \log \rho}=\frac{\beta_{M}}{\log \rho}, \quad \lim _{q \rightarrow-\infty} \frac{\tau\left(\mu_{k}, q\right)}{q}=\lim _{q \rightarrow-\infty} \frac{P_{M}(q)}{q \log \rho}=\frac{\alpha_{M}}{\log \rho}
$$

To prove (S1), we shall present some notation at first. For convenience, we denote $R\left(i_{1} \ldots i_{n}\right):=\operatorname{conv}\left(\pi_{k}\left(\Sigma_{A} \cap\left[i_{1} \ldots i_{n}\right]\right)\right)$ for each $A$-admissible word $i_{1} \ldots i_{n}$ of length $n$ with $i_{1}=1$, and call it an $n$-th net interval. By Proposition 5.1(c), $\frac{1}{C_{1}} \rho^{n} \leq \operatorname{diam}\left(R\left(i_{1} \ldots i_{n}\right)\right) \leq C_{1} \rho^{n}$ and $\frac{1}{C_{2}}\left\|M_{i_{1}} \ldots M_{i_{n}}\right\| \leq \mu_{k}\left(R\left(i_{1} \ldots i_{n}\right)\right) \leq C_{2}\left\|M_{i_{1}} \ldots M_{i_{n}}\right\|$.

Now take any $y \in \operatorname{supp} \mu_{k}$. For any integer $n>0$ and $\delta \in\left[C_{1} \rho^{n}, C_{1} \rho^{n-1}\right)$, notice that the interval $[y-\delta, y+\delta]$ contains at least one $n$-th net interval and intersects at most $\frac{2 C_{1} \rho^{n-1}}{\left(1 / C_{1}\right) \rho^{n}}+1=2\left(C_{1}\right)^{2} \rho^{-1}+1$ many different $n$-th net intervals. It follows that

$$
\frac{1}{C_{2}} \min _{i_{1} \cdots i_{n}}\left\|M_{i_{1}} \ldots M_{i_{n}}\right\| \leq \mu_{k}([y-\delta, y+\delta]) \leq C_{2}\left(2\left(C_{1}\right)^{2} \rho^{-1}+1\right) \max _{i_{1} \cdots i_{n}}\left\|M_{i_{1}} \ldots M_{i_{n}}\right\|,
$$


where the minimum and the maximum are taken over the set of $A$-admissible words of length n. Applying (2.3) and Lemma 2.3 gives $\limsup _{\delta \rightarrow 0} \frac{\log \mu_{k}([y-\delta, y+\delta])}{\log \delta}, \liminf _{\delta \rightarrow 0} \frac{\log \mu_{k}([y-\delta, y+\delta])}{\log \delta} \in$ $\left[\frac{\beta_{M}}{\log \rho}, \frac{\alpha_{M}}{\log \rho}\right]=\left[\alpha_{\min }, \alpha_{\max }\right]$. Thus we have

$$
E_{t} \neq \emptyset \Longrightarrow t \in\left[\alpha_{\min }, \alpha_{\max }\right]
$$

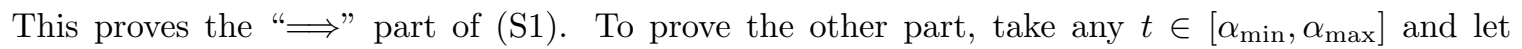
$\alpha=t \log \rho$. Then $\alpha \in\left[\alpha_{M}, \beta_{M}\right]$. Pick an $A$-admissible word $\xi=u_{1} u_{2} \ldots u_{s}$ with $u_{1}=1$ such that the net interval $R\left(u_{1} \ldots u_{s}\right)$ is strictly contained in the interior of the interval $R\left(u_{1}\right)=I_{k}$. This choice is always possible since $\mu$ contains no atoms. By Proposition 3.2, there exists a non-empty set $\Gamma=\Gamma(\xi) \subset E_{M}(\alpha)$ such that $\operatorname{dim}_{H} \Gamma=\operatorname{dim}_{H} E_{M}(\alpha)$, and for any $x=\left(x_{j}\right)_{j=1}^{\infty} \in \Gamma$, there exists a sequence of positive integers $\left\{n_{p}(x)\right\}_{p=1}^{\infty} \uparrow \infty$ such that $n_{1}(x)=1, \lim _{p \rightarrow \infty} \frac{n_{p+1}(x)}{n_{p}(x)}=1$, and $\xi$ appears in $\left(x_{j}\right)_{j=1}^{\infty}$ at each position $n_{p}(x)$. We shall finish the proof of (S1) by showing that

$$
\pi_{k}(\Gamma) \subset E_{t} \text {. }
$$

To show (5.5), let $x=\left(x_{i}\right)_{i=1}^{\infty} \in \Gamma$. Then $x_{1}=1$ and by Proposition 5.1 (b), the words $x_{1} \ldots x_{n}$ are $A$-admissible for all $n$. Notice that for each $n, \operatorname{diam}\left(R\left(x_{1} \ldots x_{n}\right)\right) \leq C_{1} \rho^{n}$, hence we have $\left[\pi_{k} x-\right.$ $\left.C_{1} \rho^{n}, \pi_{k} x+C_{1} \rho^{n}\right] \supset R\left(x_{1} \ldots x_{n}\right)$. It follows that $\mu_{k}\left(\left[\pi_{k} x-C_{1} \rho^{n}, \pi_{k} x+C_{1} \rho^{n}\right]\right) \geq \mu_{k}\left(R\left(x_{1} \ldots x_{n}\right)\right) \geq$ $\frac{1}{C_{2}}\left\|M_{x_{1}} \ldots M_{x_{n}}\right\|$. Consequently we have

$$
\begin{aligned}
\limsup _{\delta \rightarrow 0} \frac{\log \mu_{k}\left(\left[\pi_{k} x-\delta, \pi_{k} x+\delta\right]\right)}{\log \delta} & =\limsup _{n \rightarrow \infty} \frac{\log \mu_{k}\left(\left[\pi_{k} x-C_{1} \rho^{n}, \pi_{k} x+C_{1} \rho^{n}\right]\right)}{\log \left(C_{1} \rho^{n}\right)} \\
& \leq \limsup _{n \rightarrow \infty} \frac{\log \left(\frac{1}{C_{2}}\left\|M_{x_{1}} \ldots M_{x_{n}}\right\|\right)}{\log \left(C_{1} \rho^{n}\right)}=\frac{\alpha}{\log \rho}=t .
\end{aligned}
$$

To see the other direction, notice that the interval $R\left(u_{1} \ldots u_{s}\right)$ is contained in the interior of $R\left(u_{1}\right)$. Thus there exists $\gamma>0$ such that $[y-\gamma, y+\gamma] \subset R\left(u_{1}\right)$ for each $y \in R\left(u_{1} \ldots u_{s}\right)$. By Proposition $5.1(\mathrm{c} .3)$, for each $p \in \mathbb{N}$, the relative position of $R\left(x_{1} \ldots x_{n_{p}(x)} \ldots x_{n_{p}(x)+s-1}\right)$ in $R\left(x_{1} \ldots x_{n_{p}(x)}\right)$ is the same as that of $R\left(x_{n_{p}(x)} x_{n_{p}(x)+1} \ldots x_{n_{p}(x)+s-1}\right)$ in $R\left(x_{n_{p}(x)}\right)$, i.e., that of $R\left(u_{1} \ldots u_{s}\right)$ in $R\left(u_{1}\right)$. Therefore for each $y \in R\left(x_{1} \ldots x_{n_{p}(x)} \ldots x_{n_{p}(x)+s-1}\right)$,

$$
\left[y-\gamma\left|R\left(x_{1} \ldots x_{n_{p}(x)-1}\right)\right|, y+\gamma\left|R\left(x_{1} \ldots x_{n_{p}(x)-1}\right)\right|\right] \subset R\left(x_{1} \ldots x_{n_{p}(x)}\right) .
$$

Now for any large $\ell \in \mathbb{N}$, there is an integer $p$ such that $\gamma\left|R\left(x_{1} \ldots x_{n_{p+1}(x)-1}\right)\right| \leq \rho^{\ell}<\gamma\left|R\left(x_{1} \ldots x_{n_{p}(x)-1}\right)\right|$. Therefore by (5.7),

$$
\begin{aligned}
{\left[\pi_{k} x-\rho^{\ell}, \pi_{k} x+\rho^{\ell}\right] } & \subset\left[\pi_{k} x-\gamma\left|R\left(x_{1} \ldots x_{n_{p}(x)-1}\right)\right|, \pi_{k} x+\gamma\left|R\left(x_{1} \ldots x_{n_{p}(x)-1}\right)\right|\right] \\
& \subset R\left(x_{1} \ldots x_{n_{p}(x)}\right) .
\end{aligned}
$$

In the meantime,

These two inequalities imply

$$
\rho^{\ell} \geq \gamma\left|R\left(x_{1} \ldots x_{n_{p+1}(x)-1}\right)\right| \geq \frac{\gamma}{C_{1}} \rho^{n_{p+1}(x)} .
$$

$$
\frac{\log \mu_{k}\left(\left[\pi_{k} x-\rho^{\ell}, \pi_{k} x+\rho^{\ell}\right]\right)}{\log \rho^{\ell}} \geq \frac{\log \mu_{k}\left(R\left(x_{1} \ldots x_{n_{p}(x)}\right)\right)}{\log \left(\frac{\gamma}{C_{1}} \rho^{n_{p+1}(x)}\right)} \geq \frac{\log \left(C_{2}\left\|M_{x_{1}} \ldots M_{x_{n_{p}(x)}}\right\|\right)}{\log \frac{\gamma}{C_{1}}+n_{p+1}(x) \log \rho} .
$$

Let $\ell \rightarrow \infty$. Since $n_{p+1}(x) / n_{p}(x)$ tends to 1 as $p \rightarrow \infty$, we obtain

$$
\begin{aligned}
\liminf _{\delta \rightarrow 0} \frac{\log \mu_{p}\left(\left[\pi_{k} x-\delta, \pi_{k} x+\delta\right]\right)}{\log \delta}= & \liminf _{\ell \rightarrow \infty} \frac{\log \mu_{k}\left(\left[\pi_{k} x-\rho^{\ell}, \pi_{k} x+\rho^{\ell}\right]\right)}{\ell \log \rho} \\
& \geq \liminf _{p \rightarrow \infty} \frac{\log \left\|M_{x_{1}} \ldots M_{x_{n_{p}(x)}}\right\|}{n_{p}(x) \log \rho}=t .
\end{aligned}
$$


By (5.6) and (5.8), we obtain (5.5). This finishes the proof of (S1).

To show (S2), by (5.5) we have $\operatorname{dim}_{H} E_{t} \geq \operatorname{dim}_{H} \pi_{k}(\Gamma)$. Later we shall prove that

$$
\operatorname{dim}_{H} \pi_{k}(F)=\frac{\log m}{-\log \rho} \operatorname{dim}_{H} F, \quad \forall F \subset \Sigma_{A} \cap[1] .
$$

Applying (5.9) we have $\operatorname{dim}_{H} E_{t} \geq \operatorname{dim}_{H} \pi_{k}(\Gamma)=\frac{\log m}{-\log \rho} \operatorname{dim}_{H} \Gamma=\frac{\log m}{-\log \rho} \operatorname{dim}_{H} E_{M}(\alpha)$. Hence using Theorem 1.1, we have

$$
\begin{aligned}
\operatorname{dim}_{H} E_{t} & \geq \frac{1}{-\log \rho} \inf _{q \in \mathbb{R}}\left\{P_{M}(q)-\alpha q\right\} \\
& =\inf _{q \in \mathbb{R}}\left\{\frac{P_{M}(q)}{-\log \rho}-\frac{\alpha}{-\log \rho} q\right\} \\
& =\inf _{q \in \mathbb{R}}\left\{-\tau\left(\mu_{k}, q\right)+t q\right\} .
\end{aligned}
$$

However, since $E_{t} \neq \emptyset$, the upper bound $\operatorname{dim}_{H} E_{t} \leq \inf _{q \in \mathbb{R}}\left\{-\tau\left(\mu_{k}, q\right)+t q\right\}$ is generic, not depending on the special property of $\mu_{k}$ (see, e.g., [7, Theorem 1] or [31, Theorem 4.1]). This finishes the proof of (S2).

Now we prove (5.9). Actually the proof is quite standard. Since $\frac{1}{C_{1}} \rho^{n} \leq \operatorname{diam}\left(R_{i_{1} \ldots i_{n}}\right) \leq C_{1} \rho^{n}$ for any $n$-th net interval $R_{i_{1} \ldots i_{n}}$, we have

$$
\left|\pi_{k} x-\pi_{k} y\right| \leq C_{1}(d(x, y))^{\frac{-\log \rho}{\log m}}, \quad \forall x, y \in \Sigma_{A} \cap[1] .
$$

This implies (for a proof, see, e.g., [20, Proposition 2.2].)

$$
\mathcal{H}^{-s \log m / \log \rho}\left(\pi_{k} F\right) \leq\left(C_{1}\right)^{-s \log m / \log \rho} \mathcal{H}^{s}(F), \quad \forall s \geq 0, F \subset \Sigma_{A} \cap[1],
$$

where $\mathcal{H}^{u}$ denotes the $u$-dimensional Hausdorff measure. On the other hand, for each $s \geq 0$ and $F \subset \Sigma_{A} \cap[1]$, let $\left\{\Delta_{n}\right\}_{n=1}^{\infty}$ be a family of intervals such that $\operatorname{diam}\left(\Delta_{n}\right) \leq \operatorname{diam}\left(I_{k}\right), \Delta_{n} \cap \pi_{k} F \neq \emptyset$ and $\bigcup_{n \geq 1} \Delta_{n} \supset \pi_{k} F$. Then for each $n$ set

$$
G_{n}=\left\{A \text {-admissible words } i_{1} \ldots i_{p}: i_{1}=1, R_{i_{1} \ldots i_{p}} \cap \Delta_{n} \neq \emptyset, \rho^{p} \leq \operatorname{diam}\left(\Delta_{n}\right)<\rho^{p-1}\right\} .
$$

By the net property, $\# G_{n} \leq \frac{C_{1}}{\rho}+1$, and furthermore the family $\left\{\Sigma_{A} \cap[\eta]: \eta \in \bigcup_{n \geq 1} G_{n}\right\}$ is a cover of $F$ with

$$
\sum_{\eta \in \cup_{n \geq 1} G_{n}} \operatorname{diam}\left([\eta] \cap \Sigma_{A}\right)^{s} \leq\left(\frac{C_{1}}{\rho}+1\right) \sum_{n \geq 1} \operatorname{diam}\left(\Delta_{n}\right)^{-s \log m / \log \rho} .
$$

Thus we have

$$
\mathcal{H}^{-s \log m / \log \rho}\left(\pi_{k} F\right) \geq\left(C_{1} / \rho+1\right)^{-1} \mathcal{H}^{s}(F), \quad \forall s \geq 0, F \subset \Sigma_{A} \cap[1] .
$$

Combining it with (5.10) yields (5.9). This finishes the proof of Theorem 1.2.

Remark 5.3. Applying Theorem 1.2 and Proposition 5.2, we can deduce a global property of $\mu$, that is,

$$
\operatorname{dim}_{H} E_{\mu}(\alpha)=\inf _{q \in \mathbb{R}}\{\alpha q-\tau(q)\}, \quad \forall \alpha \in\left[\tau^{\prime}(+\infty), \tau^{\prime}(0-)\right],
$$

where $\tau(q)=\tau(\mu, q)$. To see this, since $\tau(q)$ is concave, we have for $\alpha \in\left[\tau^{\prime}(+\infty), \tau^{\prime}(0-)\right]$, $\inf _{q \in \mathbb{R}}\{\alpha q-\tau(q)\}=\inf _{q \geq 0}\{\alpha q-\tau(q)\}$. By Proposition 5.2, $\tau\left(\mu_{k}, q\right)=\tau(q)$ for all $q \geq 0$ and 
$k \in \Lambda$. Thus by Theorem 1.2, for $\alpha \in\left[\tau^{\prime}(+\infty), \tau^{\prime}(0-)\right]$,

$$
\begin{aligned}
\inf _{q \in \mathbb{R}}\{\alpha q-\tau(q)\} & =\inf _{q \geq 0}\{\alpha q-\tau(q)\}=\inf _{q \geq 0}\left\{\alpha q-\tau\left(\mu_{k}, q\right)\right\} \\
& =\inf _{q \in \mathbb{R}}\left\{\alpha q-\tau\left(\mu_{k}, q\right)\right\}=\operatorname{dim}_{H} E_{\mu_{k}}(\alpha) .
\end{aligned}
$$

Since $\operatorname{dim}_{H} E_{\mu}(\alpha) \geq \operatorname{dim}_{H} E_{\mu_{k}}(\alpha)$ and $\operatorname{dim}_{H} E_{\mu}(\alpha) \leq \inf _{q \in \mathbb{R}}\{\alpha q-\tau(q)\}$, the equality (5.11) follows.

Furthermore if the equality $\tau\left(\mu_{k}, q\right)=\tau(q)$ holds for all $q<0$, then we have a strong result: The measure $\mu$ itself satisfies the complete multifractal formalism. To see it, we have for all $\alpha \in$ $\left[\tau^{\prime}(+\infty), \tau^{\prime}(-\infty)\right]$

$$
\inf _{q \in \mathbb{R}}\{\alpha q-\tau(q)\}=\inf _{q \in \mathbb{R}}\left\{\alpha q-\tau\left(\mu_{k}, q\right)\right\}=\operatorname{dim}_{H} E_{\mu_{k}}(\alpha) .
$$

Since $\operatorname{dim}_{H} E_{\mu}(\alpha) \geq \operatorname{dim}_{H} E_{\mu_{k}}(\alpha)$ and $\operatorname{dim}_{H} E_{\mu}(\alpha) \leq \inf _{q \in \mathbb{R}}\{\alpha q-\tau(q)\}$, we have

$$
\operatorname{dim}_{H} E_{\mu}(\alpha)=\inf _{q \in \mathbb{R}}\{\alpha q-\tau(q)\}, \quad \forall \alpha \in\left[\tau^{\prime}(+\infty), \tau^{\prime}(-\infty)\right],
$$

On the other hand, $E_{\mu}(\alpha) \neq \emptyset$ implies $\alpha \in\left[\tau^{\prime}(+\infty), \tau^{\prime}(-\infty)\right]$. To see it, denote $a_{\epsilon}=\sup _{x \in \operatorname{supp}(\mu)} \mu([x-$ $\epsilon, x+\epsilon])$ and $b_{\epsilon}=\inf _{x \in \operatorname{supp}(\mu)} \mu([x-\epsilon, x+\epsilon])$. Denote $\tau(\epsilon, q):=\sup \sum_{i} \mu\left(B_{\epsilon}\left(x_{i}\right)\right)^{q}$, where the supremum is taken over all the families of disjoint balls $B_{\epsilon}\left(x_{i}\right)$ of radius $\epsilon$ and center $x_{i} \in \operatorname{supp}(\mu)$. Then we have

$$
\begin{cases}\left(a_{\epsilon}\right)^{q} \tau(\epsilon, q) \leq \epsilon^{-1}\left(a_{\epsilon}\right)^{q}, & \text { if } q \geq 0, \\ \left(b_{\epsilon}\right)^{q} \tau(\epsilon, q) \leq \epsilon^{-1}\left(b_{\epsilon}\right)^{q}, & \text { if } q<0 .\end{cases}
$$

Since by definition $\tau(q)=\liminf _{\epsilon \rightarrow 0} \log \tau(\epsilon, q) / \log \epsilon$, we have

$$
\begin{cases}q \liminf _{\epsilon \rightarrow 0} \frac{\log a_{\epsilon}}{\log \epsilon}-1 \leq \tau(q) \leq q \liminf _{\epsilon \rightarrow 0} \frac{\log a_{\epsilon}}{\log \epsilon}, & \text { if } q \geq 0, \\ q \lim \sup _{\epsilon \rightarrow 0} \frac{\log b_{\epsilon}}{\log \epsilon}-1 \leq \tau(q) \leq q \lim \sup _{\epsilon \rightarrow 0} \frac{\log b_{\epsilon}}{\log \epsilon} . & \text { if } q<0 .\end{cases}
$$

This implies

$$
\liminf _{\epsilon \rightarrow 0} \frac{\log a_{\epsilon}}{\log \epsilon}=\lim _{q \rightarrow+\infty} \tau(q) / q=\tau^{\prime}(+\infty), \quad \limsup _{\epsilon \rightarrow 0} \frac{\log b_{\epsilon}}{\log \epsilon}=\lim _{q \rightarrow-\infty} \tau(q) / q=\tau^{\prime}(-\infty) .
$$

Thus $E_{\mu}(\alpha) \neq \emptyset$ implies $\alpha \in\left[\tau^{\prime}(+\infty), \tau^{\prime}(-\infty)\right]$. This fact, together with (5.12), shows that $\mu$ satisfies the complete multifractal formalism.

\section{The Structure of SElF-Similar MeASURES SATISFYing the Finite type CONDition}

In this section we will outline the structure of self-similar measures satisfying the finite type condition and provide a constructive proof of Proposition 5.1. Especially we shall take the Erdös measure as an example. Almost all the results involved come from [12] and [13].

Let $S_{j} x=\rho x+b_{j}(j=1,2, \ldots, \ell)$ satisfy the finite type condition, i.e., $\left\{S_{j}\right\}$ satisfies (5.1). Let $\mu$ be the self-similar measure generated by $\left\{S_{j}\right\}_{j=1}^{\ell}$ with the probability weight $\left\{p_{j}\right\}_{j=1}^{\ell}$. Let $K$ be the corresponding self-similar set. Without loss of generality, here and afterwards we always assume

$$
0=b_{1}<b_{2}<\ldots<b_{\ell}=1-\rho .
$$

Under this assumption, the convex hull of $K$ is just the interval $[0,1]$.

Write $\mathcal{A}=\{1, \ldots, \ell\}$. For $n \in \mathbb{N}$ let $\mathcal{A}_{n}$ denote the collection of all indices $j_{1} \ldots j_{n}$ of length $n$ over $\mathcal{A}$. For $\sigma=j_{1} \ldots j_{n} \in \mathcal{A}_{n}$ write $S_{\sigma}=S_{j_{1}} \circ \ldots \circ S_{j_{n}}$. We define two families of sets $P_{n}^{0}, P_{n}^{1}(n \geq 0)$ in the following way: $P_{0}^{0}=\{0\}, P_{0}^{1}=\{1\}$, and $P_{n}^{0}=\left\{S_{\sigma}(0): \sigma \in \mathcal{A}_{n}\right\}, P_{n}^{1}=\left\{S_{\sigma}(1): \sigma \in \mathcal{A}_{n}\right\}$ 
for $n \geq 1$. Define $P_{n}=P_{n}^{0} \bigcup P_{n}^{1}$ for $n \geq 0$. Let $h_{1}, \ldots, h_{s_{n}}$ be all the elements of $P_{n}$ ranked in the increasing order. Define

$$
\mathcal{F}_{n}=\left\{\left[h_{j}, h_{j+1}\right]: 1 \leq j<s_{n},\left(h_{j}, h_{j+1}\right) \cap K \neq \emptyset\right\} .
$$

Each element in $\mathcal{F}_{n}$ is called an $n$-th basic net interval. The following facts about basic net intervals can be checked easily: (i) $\bigcup_{\Delta \in \mathcal{F}_{n}} \Delta \supset K$ for any $n \geq 0$; (ii) For any $\Delta_{1}, \Delta_{2} \in \mathcal{F}_{n}$ with $\Delta_{1} \neq \Delta_{2}$, $\operatorname{int}\left(\Delta_{1}\right) \cap \operatorname{int}\left(\Delta_{2}\right)=\emptyset$; (iii) For any $\Delta \in \mathcal{F}_{n}(n \geq 1)$, there is a unique element $\widehat{\Delta} \in \mathcal{F}_{n-1}$ such that $\widehat{\Delta} \supset \Delta$.

For each $\Delta=[a, b] \in \mathcal{F}_{n}(n \geq 0)$, we will define a positive number $\ell_{n}(\Delta)$, a vector $V_{n}(\Delta)$ and a positive integer $r_{n}(\Delta)$. If $\Delta=[0,1] \in \mathcal{F}_{0}$, we define $\ell_{0}(\Delta)=1, V_{0}(\Delta)=0$ and $r_{0}(\Delta)=1$. Otherwise for $n \geq 1$, we define $\ell_{n}(\Delta)$ and $V_{n}(\Delta)$ directly by

$$
\ell_{n}(\Delta)=\rho^{-n}(b-a)
$$

and

$$
V_{n}(\Delta)=\left(a_{1}, \ldots, a_{k}\right)
$$

where $a_{1}, \ldots, a_{k}$ (ranked in the increasing order) are all the element of the following set

$$
\left\{\rho^{-n}\left(a-S_{\sigma}(0)\right): \sigma \in \mathcal{A}_{n}, S_{\sigma}(K) \cap(a, b) \neq \emptyset\right\} .
$$

Denote by $v_{n}(\Delta)$ the dimension of $V_{n}(\Delta)$, that is, $v_{n}(\Delta)=k$. We define $r_{n}(\Delta)$ in the following way: let $\widehat{\Delta}$ be the unique interval in $\mathcal{F}_{n-1}$ containing $\Delta$, and $\Delta_{1}, \ldots, \Delta_{k}$ (ranked in the increasing order) be all the elements in $\mathcal{F}_{n}$ satisfying $\Delta_{j} \subset \widehat{\Delta}, \ell_{n}\left(\Delta_{j}\right)=\ell_{n}(\Delta), V_{n}\left(\Delta_{j}\right)=V_{n}(\Delta)$ for $1 \leq j \leq k$. Define $r_{n}(\Delta)$ to be the integer $r$ so that $\Delta_{r}=\Delta$. For convenience, we call the triple

$$
\mathcal{C}_{n}(\Delta):=\left(\ell_{n}(\Delta) ; V_{n}(\Delta) ; r_{n}(\Delta)\right)
$$

the $n$-th characteristic vector of $\Delta$, or simply characteristic vector of $\Delta$. The vector $\mathcal{C}_{n}(\Delta)$ contains the information about the length and neighborhood relation of $\Delta$. Define

$$
\Omega=\left\{\mathcal{C}_{n}(\Delta): n \geq 0, \Delta \in \mathcal{F}_{n}\right\} .
$$

For any $\alpha \in \Omega$, we write for simplicity

$$
\ell(\alpha)=\ell_{n}(\Delta), V(\alpha)=V_{n}(\Delta), v(\alpha)=v_{n}(\Delta), r(\alpha)=r_{n}(\Delta),
$$

if $\Delta \in \mathcal{F}_{n}$ and $\mathcal{C}_{n}(\Delta)=\alpha$.

Lemma 6.1. $\quad$ (i) For a given $\Delta \in \mathcal{F}_{n}(n \geq 0)$, let $\Delta_{1}, \ldots, \Delta_{k}$ (ranked in the increasing order) be all the elements in $\mathcal{F}_{n+1}$ which are subintervals of $\Delta$. Then the number $k$, the vectors $\mathcal{C}_{n+1}\left(\Delta_{i}\right)(1 \leq i \leq k)$ are determined by $\ell_{n}(\Delta)$ and $V_{n}(\Delta)$ (thus they are determined by $\left.\mathcal{C}_{n}(\Delta)\right)$

(ii) The set $\Omega$ is finite.

Proof. See [12, Lemma 2.1 and Lemma 2.3].

Let $\Omega^{*}$ denote the collection of all finite words over $\Omega$. For any $\alpha \in \Omega$, pick $n$ and $\Delta \in \mathcal{F}_{n}$ such that $\alpha=\mathcal{C}_{n}(\Delta)$. Let $\Delta_{1}, \ldots, \Delta_{k}$ (ranked in the increasing order) be all the elements in $\mathcal{F}_{n+1}$ which are subintervals of $\Delta$. Write $\alpha_{j}=\mathcal{C}_{n+1}\left(\Delta_{j}\right)$ for $1 \leq j \leq k$. By Lemma 6.1, the word $\alpha_{1} \ldots \alpha_{k}$ depend only on $\alpha$ (independent of the choice of $n$ and $\Delta$ ). We define $\zeta: \Omega \rightarrow \Omega^{*}$ by

$$
\zeta(\alpha)=\alpha_{1} \ldots \alpha_{k} .
$$

Define a $0-1$ matrix $A$ on $\Omega \times \Omega$ in the following way:

$$
A_{\alpha, \beta}= \begin{cases}1 & \text { if } \beta \text { is a letter of } \zeta(\alpha), \\ 0 & \text { otherwise. }\end{cases}
$$


A word $\beta_{1} \ldots \beta_{n} \in \Omega^{*}$ is called admissible if $A_{\beta_{j}, \beta_{j+1}}=1$ for $1 \leq j<n$.

Now we would like to associate each basic net interval with a unique admissible word. For each $\Delta \in \mathcal{F}_{n}(n \geq 0)$, we list the intervals

$$
\Delta^{0}, \Delta^{1}, \ldots, \Delta^{n}
$$

such that $\Delta^{n}=\Delta$, and $\Delta^{j}(j=0, \ldots, n-1)$ is the unique element in $\mathcal{F}_{j}$ such that $\Delta^{j} \supset \Delta^{j+1}$. The word $\alpha_{0} \alpha_{1} \ldots \alpha_{n}$, where $\alpha_{i}=\mathcal{C}_{i}\left(\Delta^{i}\right)$ for $0 \leq i \leq n$, is called the symbolic expression for $\Delta$. The introduction of the third term in a characteristic vector guarantees that two different net intervals have different symbolic expressions. Each admissible word of length $n+1$ starting from $\gamma_{0}:=\mathcal{C}_{0}([0,1])$ is a symbolic expression of some $n$-th basic net interval.

Now we analyze the distribution of $\mu$ on basic net intervals. Let $\Delta=[a, b]$ be an $n$-th basic net interval. Write $V_{n}(\Delta)=\left(a_{1}, \ldots, a_{v_{n}(\Delta)}\right)$. Iterating (5.2) $n$ times we obtain

$$
\mu(\Delta)=\sum_{\sigma \in \mathcal{A}_{n}} p_{\sigma} \mu\left(S_{\sigma}^{-1}(\Delta)\right)
$$

where $p_{\sigma}$ denotes the product $p_{j_{1}} \ldots p_{j_{n}}$ for $\sigma=j_{1} \ldots j_{n}$. Since $\mu$ is a non-atomic measure supported on $K$, we have

$$
\begin{aligned}
\mu(\Delta) & =\sum_{\substack{\sigma \in \mathcal{A}_{n}: \\
S_{\sigma}(K) \cap(a, b) \neq \emptyset}} p_{\sigma} \mu\left(S_{\sigma}^{-1}(\Delta)\right) \\
& =\sum_{i=1}^{v_{n}(\Delta)} \sum_{\sigma \in \mathcal{A}_{n}: \rho^{-n}\left(a-S_{\sigma}(0)\right)=a_{i}} p_{\sigma} \mu\left(S_{\sigma}^{-1}(\Delta)\right) \\
& =\sum_{i=1}^{v_{n}(\Delta)} \mu\left(\left[a_{i}, a_{i}+\ell_{n}(\Delta)\right]\right) \sum_{\sigma \in \mathcal{A}_{n}: S_{\sigma}(0)=a-\rho^{n} a_{i}} p_{\sigma} .
\end{aligned}
$$

Define a $v_{n}(\Delta)$-dimensional row vector $Q_{n}(\Delta)=\left(q_{1}, \ldots, q_{v_{n}(\Delta)}\right)$ by

$$
q_{i}=\mu\left(\left[a_{i}, a_{i}+\ell_{n}(\Delta)\right]\right) \sum_{\sigma \in \mathcal{A}_{n}: S_{\sigma}(0)=a-\rho^{n} a_{i}} p_{\sigma}, \quad i=1, \ldots, v_{n}(\Delta) .
$$

We call $Q_{n}(\Delta)$ the vector form of $\mu$ on $\Delta$. By $(6.4), \mu(\Delta)=\left\|Q_{n}(\Delta)\right\|:=\sum_{i=1}^{v_{n}(\Delta)} q_{i}$. Moreover, $Q_{n}(\Delta)$ is always a positive $v_{n}(\Delta)$-dimensional vector for any $n \geq 0$ and $\Delta \in \mathcal{F}_{n}$.

For any $\Delta \in \mathcal{F}_{n}(n \geq 1)$, denote by $\widehat{\Delta}$ the unique element in $\mathcal{F}_{n-1}$ so that $\widehat{\Delta} \supset \Delta$. Assume $\Delta=[a, b]$ and $\widehat{\Delta}=[c, d]$. Write $V_{n}(\Delta)=\left(a_{1}, \ldots, a_{v_{n}(\Delta)}\right)$ and $V_{n-1}(\widehat{\Delta})=\left(c_{1}, \ldots, c_{v_{n-1}(\widehat{\Delta})}\right)$. Define for any $i \in\left\{1, \ldots, v_{n}(\Delta)\right\}$ and $j \in\left\{1, \ldots, v_{n-1}(\widehat{\Delta})\right\}$,

$$
w_{j, i}= \begin{cases}p_{s} & \exists s \in \mathcal{A} \text { so that } c-\rho^{n-1} c_{j}+\rho^{n-1} b_{s}=a-\rho^{n} a_{i}, \\ 0 & \text { otherwise. }\end{cases}
$$

Define a $v_{n-1}(\widehat{\Delta}) \times v_{n}(\Delta)$ matrix $T\left(\mathcal{C}_{n-1}(\widehat{\Delta}), \mathcal{C}_{n}(\Delta)\right)=\left(t_{j, i}\right)$ by

$$
t_{j, i}=\frac{w_{j, i} \mu\left(\left[a_{i}, a_{i}+\ell_{n}(\Delta)\right]\right)}{\mu\left(\left[c_{j}, c_{j}+\ell_{n-1}(\widehat{\Delta})\right]\right)}, \quad 1 \leq j \leq v_{n-1}(\widehat{\Delta}), 1 \leq i \leq v_{n}(\Delta) .
$$

We have

$$
Q_{n}(\Delta)=Q_{n-1}(\widehat{\Delta}) T\left(\mathcal{C}_{n-1}(\widehat{\Delta}), \mathcal{C}_{n}(\Delta)\right)
$$

Since $\rho^{-n}(c-a)$ depends only on $\mathcal{C}_{n-1}(\widehat{\Delta})$ and $\mathcal{C}_{n}(\Delta)$, so does $\left(w_{j, i}\right)$. Thus $T\left(\mathcal{C}_{n-1}(\widehat{\Delta}), \mathcal{C}_{n}(\Delta)\right)$ depends only on $\mathcal{C}_{n-1}(\widehat{\Delta})$ and $\mathcal{C}_{n}(\Delta)$. Hence we have 
Proposition 6.2. ([12, Theorem 3.3]) Let $T(\alpha, \beta)\left(\alpha, \beta \in \Omega, A_{\alpha, \beta}=1\right)$ be a family of non-negative matrices defined as above, then for any $\Delta \in \mathcal{F}_{n}$,

$$
Q_{n}(\Delta)=T\left(\gamma_{0}, \gamma_{1}\right) \ldots T\left(\gamma_{n-1}, \gamma_{n}\right),
$$

where $\gamma_{0} \ldots \gamma_{n}$ is the symbolic expression of $\Delta$.

Moreover we can express precisely the entries of the product $T\left(\alpha_{1}, \alpha_{2}\right) \ldots T\left(\alpha_{n-1}, \alpha_{n}\right)$ for a given admissible word $\alpha_{1} \ldots \alpha_{n}$. To see this, choose $t \in \mathbb{N}$ and $\Delta=[a, b] \in \mathcal{F}_{t}$ so that $\mathcal{C}_{t}(\Delta)=\alpha_{1}$. Assume that the symbolic expression of $\Delta$ is $\gamma_{0} \ldots \gamma_{t-1} \alpha_{1}$. Then there is a unique $\Delta^{\prime}=[e, f] \in \mathcal{F}_{t+n-1}$ whose symbolic expression is $\gamma_{0} \ldots \gamma_{t-1} \alpha_{1} \ldots \alpha_{n}$. Write $V_{t}(\Delta)=\left(a_{1}, \ldots, a_{v_{t}(\Delta)}\right)$ and $V_{t+n-1}\left(\Delta^{\prime}\right)=$ $\left(e_{1}, \ldots, e_{v_{t+n-1}\left(\Delta^{\prime}\right)}\right)$. Denote for simplicity $X=T\left(\alpha_{1}, \alpha_{2}\right) \ldots T\left(\alpha_{n-1}, \alpha_{n}\right)$. Then from the construction of $T(\alpha, \beta)$, we have by induction that

Proposition 6.3. ([12, Proposition 3.5]) For any $1 \leq j \leq v_{t}(\Delta)$, and $1 \leq i \leq v_{t+n-1}\left(\Delta^{\prime}\right)$,

$$
X_{j, i}=\frac{\mu\left(\left[e_{i}, e_{i}+\ell_{t+n-1}\left(\Delta^{\prime}\right)\right]\right.}{\mu\left(\left[a_{j}, a_{j}+\ell_{t}(\Delta)\right]\right)} \cdot \sum_{\xi \in \mathcal{A}_{n-1}: a-\rho^{t} a_{j}+\rho^{t} S_{\xi}(0)=e-\rho^{t+n-1} e_{i}} p_{\xi} .
$$

A non-empty subset $\widehat{\Omega}$ of $\Omega$ is said to be an essential class of $\Omega$ if it satisfies: (i) $\left\{\beta \in \Omega: A_{\alpha, \beta}=\right.$ 1\} $\subset \widehat{\Omega}$ for any $\alpha \in \widehat{\Omega}$; (ii) for any $\alpha, \beta \in \widehat{\Omega}$, there exist $\gamma_{1}, \ldots, \gamma_{n} \in \widehat{\Omega}$ such that $\gamma_{1}=\alpha, \gamma_{n}=\beta$ and $A_{\gamma_{i}, \gamma_{i+1}}=1$ for $1 \leq i \leq n-1$. The existence of at least one essential class is well known (see, e.g., Lemma 1.1 of [45]). In the following we prove

Lemma 6.4. $\quad$ (i) $\Omega$ has exactly one essential class $\widehat{\Omega}$.

(ii) For any $\alpha \in \Omega, k \in\{1, \ldots, v(\alpha)\}$ and $\eta \in \widehat{\Omega}$, there exists an admissible word $\alpha_{1} \ldots \alpha_{j}$ with $j \geq 2$ such that $\alpha_{1}=\alpha, \alpha_{j}=\eta$ and all the entries of the $k$-th row of the matrix $T\left(\alpha_{1}, \alpha_{2}\right) \ldots T\left(\alpha_{j-1}, \alpha_{j}\right)$ are positive.

(iii) There exist two constants $N \in \mathbb{N}$ and $C>0$ such that for any $\Delta \in \mathcal{F}_{n}$ and any $\eta \in \widehat{\Omega}$, there exist $s \leq N$ and $\widehat{\Delta} \subset \Delta$ with $\widehat{\Delta} \in F_{n+s}$ and $\mathcal{C}_{n+s}(\widehat{\Delta})=\eta$ such that $\mu(\widehat{\Delta}) \geq C \mu(\Delta)$.

Proof. We adopt a similar method used in the proof of Lemma 4.4 in [12]. To show that $\Omega$ has exactly one essential class, it suffices to prove that there exists an element $\gamma$ in $\Omega$ such that for any $\alpha \in \Omega$, there is an admissible word beginning with $\alpha$ and ending by $\gamma$. Equivalently, one needs to show that for any $n$ and $\Delta_{1} \in \mathcal{F}_{n}$, there exists $\Delta_{2} \subset \Delta_{1}$ with $\Delta_{2} \in \mathcal{F}_{n^{\prime}}$ for some $n^{\prime}>n$ and $\mathcal{C}_{n^{\prime}}\left(\Delta_{2}\right)=\gamma$.

To achieve this, we pick $\beta \in \Omega$ such that (i) $\ell(\beta)=\min \{\ell(\eta): \eta \in \Omega\} ;$ (ii) $v(\beta)=\max \{v(\eta)$ : $\eta \in \Omega, \ell(\eta)=\ell(\beta)\}$, where $v(\cdot)$ and $\ell(\cdot)$ are defined as in (6.2). Fix an element $\gamma \in \Omega$ such that $A_{\beta, \gamma}=1$. Choose $q \in \mathbb{N}$ and $\Delta=[c, d] \in \mathcal{F}_{q}$ such that $\mathcal{C}_{q}(\Delta)=\beta$. Write $V_{q}(\Delta)=\left(c_{1}, \ldots, c_{v_{q}(\Delta)}\right)$.

Choose arbitrarily $n \in \mathbb{N}$ and $\Delta_{1}=[a, b] \in \mathcal{F}_{n}$. Write $V_{n}\left(\Delta_{1}\right)=\left(a_{1}, \ldots, a_{v_{n}\left(\Delta_{1}\right)}\right)$. Fix $k \in$ $\left\{1, \ldots, v_{n}\left(\Delta_{1}\right)\right.$. By the definition of $V_{n}\left(\Delta_{1}\right)$, there exists $\sigma \in \mathcal{A}_{n}$ with $S_{\sigma}(0)=a-\rho^{n} a_{k}$ and $S_{\sigma}(K) \cap(a, b) \neq \emptyset$. Find a large integer $l$ and $\phi \in \mathcal{A}_{l}$ so that $S_{\sigma \phi}(K) \subset(a, b)$ and thus $S_{\sigma \phi}([0,1]) \subset$ $(a, b)$, where $\sigma \phi$ denotes the concatenation of $\sigma$ and $\phi$.

Denote $\widehat{\Delta}=S_{\sigma \phi}(\Delta)$. It is clear $\widehat{\Delta} \subset(a, b)$ since $S_{\sigma \phi}([0,1]) \subset(a, b)$. We claim that $\widehat{\Delta} \in \mathcal{F}_{n+l+q}$ with $V_{n+l+q}(\widehat{\Delta})=V_{q}(\Delta)$ and $\ell_{n+l+q}(\widehat{\Delta})=\ell_{q}(\Delta)$. First we show $\widehat{\Delta} \in \mathcal{F}_{n+l+q}$ and $\ell_{n+l+q}(\widehat{\Delta})=$ $\ell_{q}(\Delta)$. To see this, we observe that the two endpoints of $\widehat{\Delta}$ belong to the set $P_{n+l+q}$ since those of $\Delta$ belong to $P_{q}$. In the meantime $\operatorname{int}(\widehat{\Delta}) \cap K \neq \emptyset$ since $\operatorname{int}(\Delta) \cap K \neq \emptyset$. Therefore, $\widehat{\Delta}$ contains at least one element in $\mathcal{F}_{n+l+q}$. On the other hand the minimality of $\ell(\beta)$ shows that each $(n+l+q)$-th 
basic net interval has length at least $\rho^{n+l+q} \ell(\beta)$, i.e., the length of $\widehat{\Delta}$. Combining these two facts we have $\widehat{\Delta} \in \mathcal{F}_{n+l+q}$ and $\ell_{n+l+q}(\widehat{\Delta})=\ell(\beta)=\ell_{q}(\Delta)$. To show $V_{n+l+q}(\widehat{\Delta})=V_{q}(\Delta)$, by the maximum of $v(\beta)$ it suffices to show each coordinate of the vector $V_{q}(\Delta)$ is a coordinate of $V_{n+l+q}(\widehat{\Delta})$. To prove this, note that for any $1 \leq u \leq v_{q}(\Delta)$, there exists $\psi \in \mathcal{A}_{q}$ such that $S_{\psi}(0)=c-\rho^{q} c_{u}$ and $S_{\psi}(K) \cap(c, d) \neq \emptyset$. Therefore, $S_{\sigma \phi \psi}(0)=S_{\sigma \phi}(c)-\rho^{n+l+q} c_{u}$ and $S_{\sigma \phi \psi}(K) \cap \operatorname{int}\left(S_{\sigma \phi}(\Delta)\right) \neq \emptyset$. Note that $\widehat{\Delta}=S_{\sigma \phi}(\Delta)$ and $S_{\sigma \phi}(c)$ is the left endpoint of $\widehat{\Delta}$. By the definition of $V_{n+l+q}(\widehat{\Delta}), c_{u}$ is a coordinate of $V_{n+l+q}(\widehat{\Delta})$. Thus the claim follows. Since the first two terms of $\mathcal{C}_{n+l+q}(\widehat{\Delta})$ are the same as $\mathcal{C}_{q}(\Delta)=\beta$ and $A_{\beta, \gamma}=1$, by Lemma 6.1, there exists an $(n+l+q+1)$-th basic net interval $\Delta_{2} \subset \widehat{\Delta}$ such that $\mathcal{C}_{n+l+q+1}\left(\Delta_{2}\right)=\gamma$. This finishes the proof of part (i) of the lemma.

To show part (ii), we use the same notation as in the above proof. Denote by $\gamma_{0} \ldots \gamma_{n-1} \alpha$ the symbolic expression of $\Delta_{1}$. Since $\widehat{\Delta} \in \mathcal{F}_{n+l+q}$ and $\widehat{\Delta} \subset \Delta_{1}$, we can denote by $\gamma_{0} \ldots \gamma_{n-1} \alpha \gamma_{n+1} \ldots \gamma_{n+l+q}$ the symbolic expression of $\widehat{\Delta}$. Especially as proved above, $\gamma_{n+l+q}$ satisfies $V\left(\gamma_{n+l+q}\right)=V(\beta)$ and $\ell\left(\gamma_{n+l+q}\right)=\ell(\beta)$. Denote

$$
X=T\left(\alpha, \gamma_{n+1}\right) T\left(\gamma_{n+1}, \gamma_{n+2}\right) \ldots T\left(\gamma_{n+l+q-1}, \gamma_{n+l+q}\right) .
$$

By Proposition 6.3, for any $1 \leq u \leq v(\beta)=v\left(\gamma_{n+l+q}\right)$,

$$
X_{k, u}=\frac{\mu\left(\left[c_{u}, c_{u}+\ell(\beta)\right]\right)}{\mu\left(\left[a_{k}, a_{k}+\ell(\alpha)\right]\right)} . \sum_{\xi \in \mathcal{A}_{l+q}: a-\rho^{n} a_{k}+\rho^{n} S_{\xi}(0)=S_{\sigma \phi}(c)-\rho^{n+l+q} c_{u}} p_{\xi} .
$$

Recall that we have proved in last paragraph that for each $1 \leq u \leq v(\beta)$, there exists $\psi \in \mathcal{A}_{q}$ such that $S_{\sigma \phi \psi}(0)=S_{\sigma \phi}(c)-\rho^{n+l+q} c_{u}$. Note that

$$
S_{\sigma \phi \psi}(0)=S_{\sigma}(0)+\rho^{n} S_{\phi \psi}(0)=a-\rho^{n} a_{k}+\rho^{n} S_{\phi \psi}(0) .
$$

By (6.6), $X_{k, u}>0$. Therefore

$$
\mathbf{e}_{k} T\left(\alpha, \gamma_{n+1}\right) T\left(\gamma_{n+1}, \gamma_{n+2}\right) \ldots T\left(\gamma_{n+l+q-1}, \gamma_{n+l+q}\right)>\mathbf{0},
$$

where $\mathbf{e}_{k}$ denotes the $v(\alpha)$-dimensional row vector whose $k$-th coordinate is 1 and all other coordinates are 0 .

Choose an admissible sequence $\eta_{1} \ldots \eta_{t}$ such that $\eta_{1}=\gamma$ and $\eta_{t}=\eta$. By (6.7),

$$
\mathbf{e}_{k} T\left(\alpha, \gamma_{n+1}\right) \ldots T\left(\gamma_{n+l+q-1}, \gamma_{n+l+q}\right) T\left(\gamma_{n+l+q}, \eta_{1}\right) T\left(\eta_{1}, \eta_{2}\right) \ldots T\left(\eta_{t-1}, \eta_{t}\right)>\mathbf{0},
$$

That is, all the entries of the $k$-th row of the matrix

$$
T\left(\alpha, \gamma_{n+1}\right) T\left(\gamma_{n+1}, \gamma_{n+2}\right) \ldots T\left(\gamma_{n+l+q-1}, \gamma_{n+l+q}\right) T\left(\gamma_{n+l+q}, \eta_{1}\right) T\left(\eta_{1}, \eta_{2}\right) \ldots T\left(\eta_{t-1}, \eta_{t}\right)
$$

are positive, which completes the proof of part (ii).

Part (iii) follows from part (ii). To see it, by part (ii) we can construct a finite family of admissible words $\left\{\alpha_{1} \ldots \alpha_{j}\right\}_{\alpha, k, \eta}$ when $\alpha, k$ and $\eta$ are taken over the set $\Omega,\{v(1), \ldots, v(\alpha)\}$ and $\widehat{\Omega}$ respectively. Let $N$ denote the largest length of the words in this family. Let $D$ denote the smallest non-zero entry appear in the constructed matrices $T\left(\alpha_{1}, \alpha_{2}\right) \ldots T\left(\alpha_{j-1}, \alpha_{j}\right)$.

Now fix $\Delta \in \mathcal{F}_{n}$ and $\eta \in \widehat{\Omega}$. Assume $\mathcal{C}_{n}(\Delta)=\alpha$. Choose $k \in\{1, \ldots, v(\alpha)\}$ such that $k$-th entry of $Q_{n}(\Delta)$ is larger than or equals to $\frac{1}{v(\alpha)}\left\|Q_{n}(\Delta)\right\|=\frac{1}{v(\alpha)} \mu(\Delta)$. Let $\alpha_{1} \ldots \alpha_{j}$ be a constructed admissible word beginning with $\alpha$ and ending by $\eta$, such that the $k$-th row of the matrix $T\left(\alpha_{1}, \alpha_{2}\right) \ldots T\left(\alpha_{j-1}, \alpha_{j}\right)$ are positive. Let $W$ be the symbolic expression of $\Delta$. Then there exists a unique one $\widehat{\Delta} \subset \Delta$ with $\widehat{\Delta} \in \mathcal{F}_{n+j-1}$ such that symbolic expression of $\widehat{\Delta}$ is $W \alpha_{2} \ldots \alpha_{j}$. Then

$$
\mu(\widehat{\Delta})=\left\|Q_{n}(\Delta) T\left(\alpha_{1}, \alpha_{2}\right) \ldots T\left(\alpha_{j-1}, \alpha_{j}\right)\right\| \geq \frac{1}{D v(\alpha)} \mu(\Delta) .
$$


This finishes the proof by letting $C=\inf _{\alpha \in \Omega} \frac{1}{D v(\alpha)}$.

Let $\widehat{\Omega}$ be the essential class of $\Omega$. In what follows we always denote $m:=\# \widehat{\Omega}$ and write $\widehat{\Omega}=$ $\left\{\eta_{1}, \ldots, \eta_{m}\right\}$. Set $d=\sum_{i=1}^{m} v\left(\eta_{i}\right)$, where $v(\cdot)$ is defined as in (6.2). In the following we construct a family of $d \times d$ matrices $\left\{M_{i}\right\}_{i=1}^{m}$. For any $1 \leq i \leq m$, define $M_{i}$ to be the partitioned matrix

$$
M_{i}=\left[\begin{array}{cccc}
U_{1,1}^{i} & U_{1,2}^{i} & \cdots & U_{1, m}^{i} \\
U_{2,1}^{i} & U_{2,2}^{i} & \cdots & U_{2, m}^{i} \\
\vdots & \vdots & \ddots & \vdots \\
U_{m, 1}^{i} & U_{m, 2}^{i} & \cdots & U_{m, m}^{i}
\end{array}\right],
$$

where for each $1 \leq j, k \leq m, U_{j, k}^{i}$ is a $v\left(\eta_{j}\right) \times v\left(\eta_{k}\right)$ matrix defined by

$$
U_{j, k}^{i}= \begin{cases}T\left(\eta_{j}, \eta_{i}\right) & \text { if } k=i \text { and } A_{\eta_{j}, \eta_{i}}=1 \\ \mathbf{0} & \text { otherwise. }\end{cases}
$$

Let $J_{0} \in \mathcal{F}_{n_{0}}$ so that $\mathcal{C}_{n_{0}}\left(J_{0}\right)=\eta_{1}$. Denote $\Theta_{0} \in \Omega^{*}$ by $\Theta_{0}=\gamma_{0} \ldots \gamma_{n_{0}-1} \eta_{1}$ the symbolic expression of $J_{0}$. Given $\Delta \in \mathcal{F}_{n}\left(n \geq n_{0}\right)$ with $\Delta \subset J_{0}$, define $\widehat{Q}_{n}(\Delta)$ to be the partitioned vector $\left(W_{1}, \ldots, W_{m}\right)$, where $W_{i}$ is a $v\left(\eta_{i}\right)$-dimensional row vector defined by

$$
W_{i}= \begin{cases}Q_{n}(\Delta) & \text { if } \eta_{i}=\mathcal{C}_{n}(\Delta) \\ \mathbf{0} & \text { otherwise }\end{cases}
$$

It is clear that $\widehat{Q}_{n}(\Delta)$ is a $d$-dimensional row vector, which is called the uniform vector form of $\mu$ on $\Delta$. We have

Lemma 6.5. (i) The matrix $H:=\sum_{i=1}^{m} M_{i}$ is irreducible. That is, there exists an integer $r>0$ such that $H^{r}>\mathbf{0}$.

(ii) Given $\Delta \in \mathcal{F}_{n_{0}+n}(n \geq 1)$ with $\Delta \subset J_{0}$, we have

$$
\widehat{Q}_{n_{0}+n}(\Delta)=\widehat{Q}_{n_{0}}\left(J_{0}\right) M_{i_{1}} \ldots M_{i_{n}} \approx \mathbf{e} M_{1} M_{i_{1}} \ldots M_{i_{n}},
$$

where $\Theta_{0} \eta_{i_{1}} \ldots \eta_{i_{k}}$ is the symbolic expression of $\Delta$, $\mathbf{e}$ denotes the d-dimensional row vector of which each entry equals 1 , and the constant involved in " $\approx$ " only depends on $J_{0}$. Hence $\mu(\Delta) \approx\left\|M_{1} M_{i_{1}} \ldots M_{i_{n}}\right\|$.

(iii) $M_{i_{1}} \ldots M_{i_{k}} \neq \mathbf{0}$ if and only if $\eta_{i_{1}} \ldots \eta_{i_{k}}$ is an admissible sequence.

Proof. See [12, Lemma 4.1 and Proposition 4.2].

Proof of Proposition 5.1. Let $m, d$ and $M_{i}(1 \leq i \leq m)$ be constructed as above. Without confusion we define an $m \times m 0-1$ matrix $A=\left(A_{i, j}\right)_{1 \leq i, j \leq m}$ by

$$
A_{i, j}=A_{\eta_{i}, \eta_{j}},
$$

where $A_{\alpha, \beta}(\alpha, \beta \in \Omega)$ is defined as in (6.3). Since $\widehat{\Omega}$ is an essential class of $\Omega$, the matrix $\left(A_{i, j}\right)_{1 \leq i, j \leq m}$ is irreducible. Then part (a) and (b) of Proposition 5.1 follows from Lemma 6.5 (i) and (ii). Define $\Sigma=\{1, \ldots, m\}^{\mathbb{N}}$ and $\Sigma_{A}=\left\{\left(x_{i}\right)_{i=1}^{\infty} \in \Sigma: A_{x_{i}, x_{i+1}}=1\right.$ for $\left.i \geq 1\right\}$. Moreover we define $\Lambda \subset \Omega^{*}$ by

$\Lambda=\left\{A\right.$-admissible word $\alpha_{0} \alpha_{1} \ldots \alpha_{n}: \alpha_{0}=\gamma_{0}, \alpha_{n}=\eta_{1}, \alpha_{i} \neq \eta_{1}$ for $\left.1 \leq i<n\right\}$.

For any $W=\alpha_{0} \alpha_{1} \ldots \alpha_{n} \in \Lambda$, we denote by $I_{W}$ the $n$-th basic net interval whose symbolic expression is $W=\alpha_{0} \alpha_{1} \ldots \alpha_{n}$. By the definition of $\Lambda$ and the structure of basic net intervals, $\operatorname{int}\left(I_{W}\right) \cap$ $\operatorname{int}\left(I_{W^{\prime}}\right)=\emptyset$ for different words $W, W^{\prime} \in \Lambda$. 
Now we show $\mu\left(\bigcup_{W \in \Lambda} I_{W}\right)=1$ by contradiction. Assume that $\mu\left(\bigcup_{W \in \Lambda}\right)=t<1$. Then for any $\epsilon>0$, there exists $n \in \mathbb{N}$ such that

$$
t-\epsilon \leq \sum_{W \in \Lambda,|W| \leq n} \mu\left(I_{W}\right) \leq t
$$

where $|W|$ denotes the length of the word $W$. Let $\mathcal{F}_{n}^{\prime}$ denotes the collection of all $n$-th basic net intervals that have no intersection with the interior of $\bigcup_{W \in \Lambda,|W| \leq n} I_{W}$. Then $\bigcup_{\Delta \in \mathcal{F}_{n}^{\prime}} \cup\left(\bigcup_{W \in \Lambda,|W| \leq n} I_{W}\right) \supset$ $K$, thus $\sum_{\Delta \in \mathcal{F}_{n}^{\prime}} \mu(\Delta) \geq 1-t$. By Lemma 6.4 (iii), for any $\Delta \in \mathcal{F}_{n}^{\prime}$ there exists a corresponding $U \in \Lambda$ with $|U|^{n}>n$ such that $I_{U} \subset \Delta$ and $\mu\left(I_{U}\right) \geq C \mu(\Delta)$ for a constant $C>0$ independent of $n$. Therefore

$$
\sum_{U \in \Lambda,|U|>n} \mu\left(I_{U}\right) \geq C \sum_{\Delta \in \mathcal{F}_{n}^{\prime}} \mu(\Delta) \geq C(1-t),
$$

which leads to a contradiction with the fact $\sum_{U \in \Lambda,|U|>n} \mu\left(I_{U}\right) \leq \epsilon$, since $\epsilon$ can be taken arbitrarily small.

Now for $W=\alpha_{0} \alpha_{1} \ldots \alpha_{n} \in \Lambda$ we define the map $\pi_{W}: \Sigma_{A} \cap[1] \rightarrow K \cap I_{W}$ by

$$
\pi_{W}(x)=\bigcap_{k=2}^{\infty} \Delta_{x_{1} x_{2} \ldots x_{k}}^{W}, \quad \forall x=\left(x_{i}\right)_{i=1}^{\infty},
$$

where $\Delta_{x_{1} x_{2} \ldots x_{k}}^{W}$ denotes the basic net interval whose symbolic expression is $W \eta_{x_{2}} \ldots \eta_{x_{k}}$. By the structure of basic net intervals, $\operatorname{conv}\left(\pi_{W}\left(\Sigma_{A} \cap\left[x_{1} x_{2} \ldots x_{k}\right]\right)=\Delta_{x_{1} x_{2} \ldots x_{k}}^{W}\right.$. From this fact and Lemma 6.5 (ii), part (c) of Proposition 5.1 follows. This finishes the proof.

Example 6.6. Let $\rho=\frac{\sqrt{5}-1}{2}$ and let $\mu$ be the self-similar measure generated by the family of maps $\left\{S_{1} x=\rho x, S_{2} x=\rho x+1-\rho\right\}$ and the probability weight $\{1 / 2,1 / 2\}$. The measure $\mu$, which is also called the Erdös measure, is the well known Bernoulli convolution associated with the golden ratio. The structure of this measure has been extensively studied in [13]. For this measure we have $\Omega=\left\{\alpha_{1}, \alpha_{2}, \ldots, \alpha_{7}\right\}$, where

$$
\begin{aligned}
& \alpha_{1}=(1 ; 0 ; 1), \quad \alpha_{2}=(\rho ; 0 ; 1), \quad \alpha_{3}=(1-\rho ;(0, \rho) ; 1), \quad \alpha_{4}=(\rho ; 1-\rho ; 1), \\
& \alpha_{5}=(\rho ;(0,1-\rho) ; 1), \quad \alpha_{6}=(2 \rho-1 ; 1-\rho ; 1), \quad \alpha_{7}=(1-\rho ;(0, \rho) ; 2) .
\end{aligned}
$$

The map $\zeta: \Omega \rightarrow \Omega^{*}$ is given by

$$
\begin{array}{ll}
\zeta\left(\alpha_{1}\right)=\alpha_{2} \alpha_{3} \alpha_{4}, & \zeta\left(\alpha_{2}\right)=\alpha_{2} \alpha_{3}, \quad \zeta\left(\alpha_{3}\right)=\alpha_{5}, \quad \zeta\left(\alpha_{4}\right)=\alpha_{3} \alpha_{4}, \\
\zeta\left(\alpha_{5}\right)=\alpha_{3} \alpha_{6} \alpha_{7}, & \zeta\left(\alpha_{6}\right)=\alpha_{3}, \quad \zeta\left(\alpha_{7}\right)=\alpha_{5} .
\end{array}
$$

Let $A$ be defined as (6.3). The matrices $\left\{T(\alpha, \beta): \alpha, \beta \in \Omega, A_{\alpha, \beta}=1\right\}$ are given by

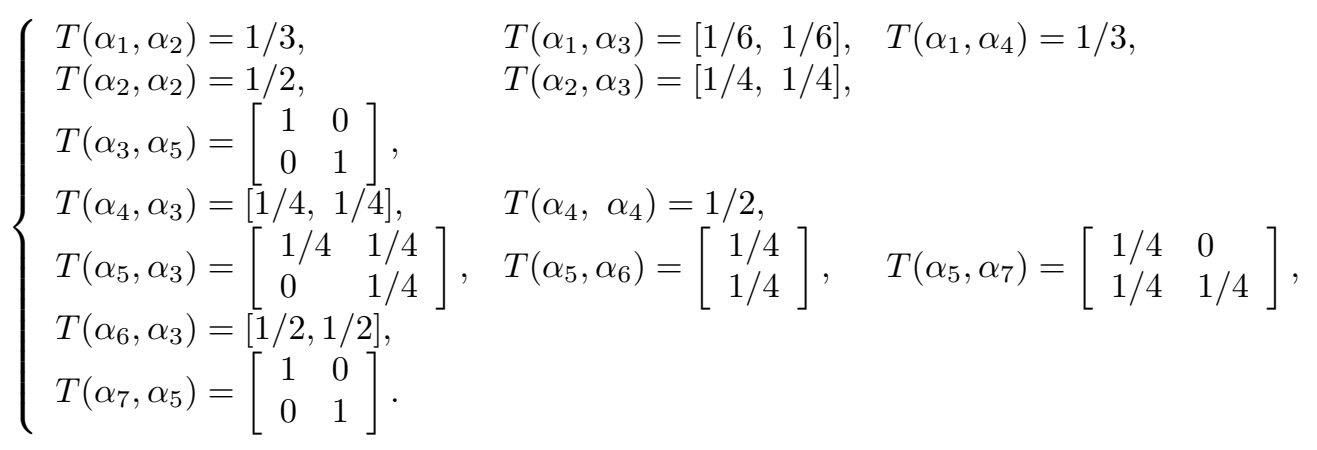


The essential class of $\Omega$ is $\widehat{\Omega}=\left\{\alpha_{3}, \alpha_{5}, \alpha_{6}, \alpha_{7}\right\}$. For convenience we relabel the elements of $\widehat{\Omega}$ by

$$
1:=\alpha_{3}, \quad 2:=\alpha_{5}, \quad 3:=\alpha_{6}, \quad 4:=\alpha_{7} .
$$

The matrices $M_{i}(1 \leq i \leq 4)$ are given by

$$
\begin{aligned}
& M_{1}=\left[\begin{array}{ccccccc}
0 & 0 & 0 & 0 & 0 & 0 & 0 \\
0 & 0 & 0 & 0 & 0 & 0 & 0 \\
1 / 4 & 1 / 4 & 0 & 0 & 0 & 0 & 0 \\
0 & 1 / 4 & 0 & 0 & 0 & 0 & 0 \\
1 / 2 & 1 / 2 & 0 & 0 & 0 & 0 & 0 \\
0 & 0 & 0 & 0 & 0 & 0 & 0 \\
0 & 0 & 0 & 0 & 0 & 0 & 0
\end{array}\right], \quad M_{2}=\left[\begin{array}{lllllll}
0 & 0 & 1 & 0 & 0 & 0 & 0 \\
0 & 0 & 0 & 1 & 0 & 0 & 0 \\
0 & 0 & 0 & 0 & 0 & 0 & 0 \\
0 & 0 & 0 & 0 & 0 & 0 & 0 \\
0 & 0 & 0 & 0 & 0 & 0 & 0 \\
0 & 0 & 1 & 0 & 0 & 0 & 0 \\
0 & 0 & 0 & 1 & 0 & 0 & 0
\end{array}\right], \\
& M_{3}=\left[\begin{array}{ccccccc}
0 & 0 & 0 & 0 & 0 & 0 & 0 \\
0 & 0 & 0 & 0 & 0 & 0 & 0 \\
0 & 0 & 0 & 0 & 1 / 4 & 0 & 0 \\
0 & 0 & 0 & 0 & 1 / 4 & 0 & 0 \\
0 & 0 & 0 & 0 & 0 & 0 & 0 \\
0 & 0 & 0 & 0 & 0 & 0 & 0 \\
0 & 0 & 0 & 0 & 0 & 0 & 0
\end{array}\right], \quad M_{4}=\left[\begin{array}{lllllcc}
0 & 0 & 0 & 0 & 0 & 0 & 0 \\
0 & 0 & 0 & 0 & 0 & 0 & 0 \\
0 & 0 & 0 & 0 & 0 & 1 / 4 & 0 \\
0 & 0 & 0 & 0 & 0 & 1 / 4 & 1 / 4 \\
0 & 0 & 0 & 0 & 0 & 0 & 0 \\
0 & 0 & 0 & 0 & 0 & 0 & 0 \\
0 & 0 & 0 & 0 & 0 & 0 & 0
\end{array}\right] .
\end{aligned}
$$

For this special case the $L^{q}$-spectrum $\tau(\mu, q)$ of $\mu$ can be expressed explicitly $[13,30]$ and has a non-differentiable point $q_{0}<0$ [13]. In fact by using the same idea in [13], one can get the formula for $\tau\left(\mu_{k}, q\right)$ and prove $\tau\left(\mu_{k}, q\right)=\tau(q)$ for all $q \in \mathbb{R}$ and $k \in \Lambda$. Since $P_{M}(q)=(\log \rho) \tau\left(\mu_{k}, q\right)$ for $q \in \mathbb{R}, P_{M}(q)$ has a non-differentiable point at $q_{0}$.

\section{REFERENCES}

[1] L. Barreira and B. Saussol, Variational principles and mixed multifractal spectra, Trans. Amer. Math. Soc. 353 (2001), 3919-3944.

[2] L. Barreira, B. Saussol and J. Schmeling, Higher-dimensional multifractal analysis, J. Math. Pures. Appl. 81 (2002), 67-91.

[3] L. Barreira and J. Schmeling, Sets of "non-typical" points have full topological entropy and full Hausdorff dimension, Israel J. Math. 116 (2000), 29-70.

[4] F. Ben Nasr, I. Bhouri and Y. Heurteaux, The validity of the multifractal formalism: results and examples, Adv. Math. 165 (2002), 264-284.

[5] P. Billingsley, Ergodic theory and information, John Wiley, New York, 1965.

[6] P. Bougerol and J. Lacroix, Products of random matrices with applications to Schrdinger operators, Birkhäuser, Boston, 1985.

[7] G. Brown, G. Michon and J. Peyrière, On the multifractal analysis of measures, J. Statist. Phys. 66 (1992), 775-790.

[8] R. Cawley and R. D. Mauldin, Multifractal decompositions of Moran fractals, Adv. Math. 92 (1992), $196-236$.

[9] A. H. Fan and D. J. Feng, On the distribution of long-term time average on the symbolic space, $J$. Stat. Phys., 99 (2000), 813-856.

[10] A. H. Fan, D. J. Feng and J. Wu, Recurrence, dimension and entropy, J. Lond. Math. Soc. 64 (2001), $229-244$.

[11] D. J. Feng, Lyapunov exponents for products of matrices and multifractal analysis, Part I: Positive matrices, Israel J. Math. 138 (2003), 353-376.

[12] D. J. Feng, Smoothness of the $L^{q}$-spectrum of self-similar measures with overlaps, J. London Math. Soc. 68 (2003), 102-118. 
[13] D. J. Feng, The limited Rademacher functions and Bernoulli convolutions associated with Pisot numbers, Adv. Math. 195 (2005), 24-101.

[14] D. J. Feng and K. S. Lau, The pressure function for products of non-negative matrices, Math. Res. Lett. 9 (2002), 363-378.

[15] D. J. Feng, K. S. Lau and X. Y. Wang, Some exceptional phenomena in multifrcatal formalism, Asian J. Math. 9 (2005), 473-488.

[16] D. J. Feng, K. S. Lau and J. Wu, Ergodic limits on the conformal repellers, Adv. Math. 169 (2002), 58-91.

[17] D. J. Feng and E. Olivier, Multifractal analysis of the weak Gibbs measures and phase transitionapplication to some Bernoulli convolutions, Ergodic Theory Dynam. System 23 (2003), 1751-1784.

[18] H. Furstenberg and H. Kesten, Products of random matrices, Ann. Math. Stat. 31 (1960), 457-469.

[19] P. Mattila, Geometry of sets and measures in Euclidean spaces, Cambridge University Press, Cambridge, 1995.

[20] K. J. Falconer, Fractal Geometry-Mathematical Foundations and Applications, John Wiley \& Sons, Chichester, 1990.

[21] K. J. Falconer, Techniques in fractal geometry, John Wiley \& Sons, Chichester, 1997.

[22] K. Falconer and A. Sloan, Continuity of subadditive pressure for self-affine sets, Real Analysis Exchange. (to appear)

[23] K. Falconer and A. Sloan, Multifractal analysis of Lyapunov exponents for general linear mappings. Preprint

[24] U. Frisch and G. Parisi, Fully developped turbulence and intermittency, Proc. Internat. School Phys. Enrico Fermi 84-88, North Holland, 1985.

[25] T. C. Hasley, M. H. Jensen, L. P. Kadanoff, I. Procaccia and B. J. Shraiman, Fractal measures and their singularities: The characterization of strange sets, Phys. Rev. A 33 (1986), 1141-1151.

[26] Y. Heurteaux, Estimations de la dimension inférieure et de la dimension supérieure des mesures, Ann. Inst. H. Poincaré Probab. Statist. 34 (1998), 309-338.

[27] T. Y. Hu and K. S. Lau, Multifractal structure of convolution of the Cantor measure, Adv. in Appl. Math. 27 (2001), 1-16.

[28] J. E. Hutchinson, Fractals and self-similarity, Indiana Univ. Math. J. 30 (1981), 713-747.

[29] K. S. Lau, Iterated function systems with overlaps and multifractal structure, Trends in probability and related analysis (Taipei, 1998), 35-76, World Sci. Publishing, River Edge, 1999.

[30] K. S. Lau and S. M. Ngai, $L^{q}$-spectrum of the Bernoulli convolution associated with the golden ratio, Studia Math. 131 (1998), 225-251.

[31] K. S. Lau and S. M. Ngai, Multifractal measures and a weak separation condition, Adv. Math. 141 (1999), 45-96.

[32] K. S. Lau and X. Y. Wang, Some exceptional phenomena in multifrcatal formalism, Part I, Asian J. Math. 9 (2005), 275-294

[33] S. M. Ngai and Y. Wang, Hausdorff dimension of self-similar sets with overlaps, J. London Math. Soc. 63 (2001), 655-672.

[34] N. T. Nguyen, Iterated function systems of finite type and the weak separation property, Proc. Amer. Math. Soc. 130 (2002), 483-487.

[35] E. Olivier, Multifractal analysis in symbolic dynamics and distribution of pointwise dimension for g-measures, Nonlinearity 12 (1999), 1571-1585.

[36] E. Olivier, N. Sidorov and A. Thomas, On the Gibbs properties of Bernoulli convolutions related to $\beta$-numeration in multinacci bases, Monatsh. Math. 145 (2005), 145-174.

[37] L. Olsen, A multifractal formalism, Adv. Math. 116 (1995), 82-196.

[38] L. Olsen and S. Winter, Normal and non-normal points of self-similar sets and divergence points of self-similar measures, J. London Math. Soc. 67 (2003), 103-122.

[39] Y. B. Pesin, Dimension theory in dynamical systems, University of Chicago Press, Chicago, 1997.

[40] Y. Pesin and H. Weiss, A multifractal analysis of Gibbs measures for conformal expanding maps and Markov Moran geometry constructions, J. Stat. Phys. 86 (1997), 233-275. 
[41] M. Pollicott, H. Weiss, Multifractal analysis of Lyapunov exponent for continued fraction and Manneville-Pomeau transformations and applications to Diophantine approximation, Comm. Math. Phys. 207 (1999), 145-171.

[42] J. Peyrière, A vectorial multifractal formalism. Fractal geometry and applications: a jubilee of Benoit Mandelbrot, Part 2, 217-230, Proc. Sympos. Pure Math., 72, Part 2, Amer. Math. Soc., Providence, RI, 2004.

[43] A. Porzio, On the regularity of the multifractal spectrum of Bernoulli convolutions, J. Statist. Phys. 91 (1998), 17-29.

[44] R. H. Riedi and B. B. Mandelbrot, Inversion formula for continuous multifractals, Adv. in Appl. Math. 19 (1997), 332-354.

[45] E. Seneta, Non-negative matrices. An introduction to theory and applications, Halsted Press, New York, 1973.

[46] P. Shmerkin, A modified multifractal formalism for a class of self-similar measures with overlap, Asian J. Math. 9 (2005), 323-348.

[47] F. Takens and E. Verbitskiy, On the variational principle for the toplogical entropy of certain noncompact sets, Ergodic Theory Dynam. System 23 (2003), 317-348.

[48] B. Testud, Phase transitions for the multifractal analysis of self-similar measures, Nonlinearity 19 (2006), 1201-1217.

[49] B. Testud, Mesures quasi-Bernoulli au sens faible: resultats et exemples, Ann. Inst. H. Poincar Probab. Statist. 42 (2006), 1-35.

[50] L. S. Young, Dimension, entropy and Lyapunov exponents, Ergodic Theory Dynam. System 2 (1982), 109-124.

Department of Mathematics, The Chinese University of Hong Kong, Shatin, Hong Kong,

E-mail address: djfeng@math.cuhk.edu.hk 